\title{
Force-Induced Unravelling of DNA Origami
}

\author{
Megan C. Engel, $*^{\dagger} \dagger$ David M. Smith, ${ }^{\ddagger}$ Markus A. Jobst, ${ }^{\S}$ Martin Sajfutdinow, ${ }^{\ddagger}$ Tim Liedl, ${ }^{\S}$ \\ Flavio Romano, Lorenzo Rovigatti, ${ }^{\dagger, \perp, \otimes \infty}$ Ard A. Louis, ${ }^{\dagger}$ and Jonathan P. K. Doye ${ }^{\#(0)}$ \\ ${ }^{\dagger}$ Rudolf Peierls Centre for Theoretical Physics, University of Oxford, 1 Keble Road, Oxford OX1 3NP, United Kingdom \\ ${ }^{\ddagger}$ Fraunhofer Institute for Cell Therapy and Immunology IZI, Perlickstra $\beta$ e 1, 04103 Leipzig, Germany \\ ${ }^{\S}$ Department für Physik, Ludwig-Maximilians-Universität Amalienstrasse 5480799 München, Germany \\ "Dipartimento di Scienze Molecolari e Nanosistemi, Università Ca' Foscari di Venezia, Via Torino 155, 30172 Venezia Mestre, Italy \\ ${ }^{\perp}$ CNR-ISC, Uos Sapienza, Piazzale A. Moro 2, 00185 Roma, Italy \\ ${ }^{\otimes}$ Dipartimento di Fisica, Sapienza Università di Roma, Piazzale A. Moro 2, 00185 Roma, Italy \\ \#Physical and Theoretical Chemistry Laboratory, Department of Chemistry, University of Oxford, South Parks Road, Oxford OX1 \\ 3QZ, United Kingdom
}

\section{Supporting Information}

\begin{abstract}
The mechanical properties of DNA nanostructures are of widespread interest as applications that exploit their stability under constant or intermittent external forces become increasingly common. We explore the force response of DNA origami in comprehensive detail by combining AFM single molecule force spectroscopy experiments with simulations using oxDNA, a coarsegrained model of DNA at the nucleotide level, to study the unravelling of an iconic origami system: the Rothemund tile. We contrast the force-induced melting of the tile with

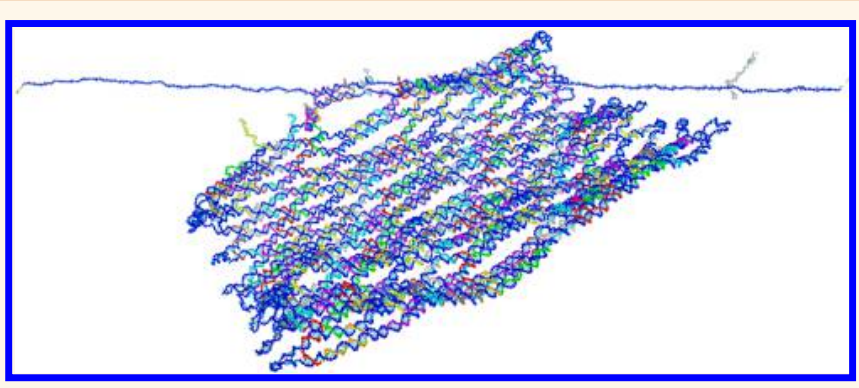
simulations of an origami 10-helix bundle. Finally, we simulate a recently proposed origami biosensor, whose function takes advantage of origami behavior under tension. We observe characteristic stick-slip unfolding dynamics in our forceextension curves for both the Rothemund tile and the helix bundle and reasonable agreement with experimentally observed rupture forces for these systems. Our results highlight the effect of design on force response: we observe regular, modular unfolding for the Rothemund tile that contrasts with strain-softening of the 10-helix bundle which leads to catastropic failure under monotonically increasing force. Further, unravelling occurs straightforwardly from the scaffold ends inward for the Rothemund tile, while the helix bundle unfolds more nonlinearly. The detailed visualization of the yielding events provided by simulation allows preferred pathways through the complex unfolding free-energy landscape to be mapped, as a key factor in determining relative barrier heights is the extensional release per base pair broken. We shed light on two important questions: how stable DNA nanostructures are under external forces and what design principles can be applied to enhance stability.
\end{abstract}

KEYWORDS: DNA nanotechnology, DNA origami, self-assembly, molecular dynamics, coarse-grained modeling, single molecule force spectroscopy, $A F M$

$\mathrm{T}$ he behavior of DNA under tension has been studied extensively both theoretically and experimentally. Single-molecule force studies have been employed to elucidate the elastic properties of double- and single-stranded DNA $;^{1-3}$ to characterize the mechanisms of duplex instability at higher forces; ${ }^{4-9}$ and to examine torsional buckling of the helix under a mixture of torque and tension. ${ }^{10,11}$ These explorations have illuminated the cellular processes that mediate structural changes in DNA, including gene regulation, transcription, and recombination. ${ }^{12-14}$ Furthermore, the now well-understood behavior of duplex DNA under force has been exploited for a wide range of applications, including employing
DNA in molecular force sensors ${ }^{15}$ to characterize mechanosensitive receptors ${ }^{16}$ and cellular traction forces. ${ }^{17}$

Denaturing of the DNA helix is typically modeled as a thermally activated process in which the dissociation rate is governed by the height of the free energy barrier between the fully zippered state-stabilized by hydrogen bonding and base stacking-and a transition state characterized by a critical number of broken base pairs. ${ }^{18,19}$ Application of an external biasing force favors more extended configurations, lowering the

Received: March 11, 2018

Accepted: May 31, 2018

Published: May 31, 2018 


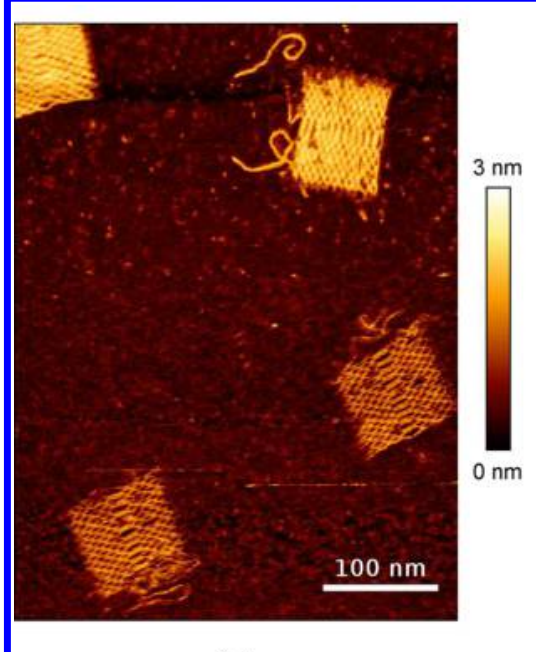

(a)

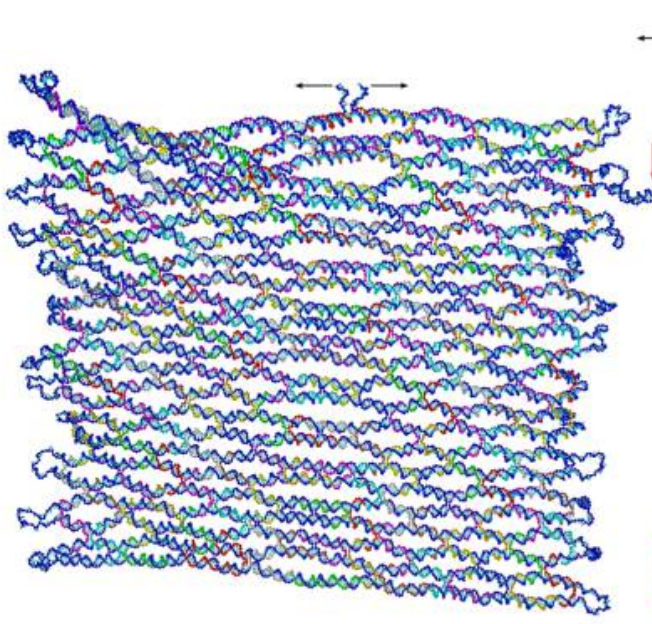

(b)

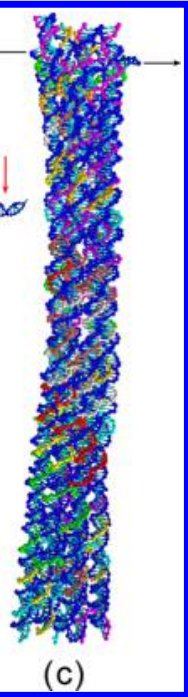

Figure 1. Two main systems studied in this work. (a) AFM image of Rothemund tiles deposited on a surface. (b) oxDNA representation of the Rothemund tile. The $20 \mathrm{bp}$-stem hairpin indicated by the red arrow was excised in Rothemund's original origami design. ${ }^{34}$ (c) oxDNA representation of the 10-helix bundle presented in Bae et al. ${ }^{64}$ For both simulated systems, forces were applied to the nucleotides at the ends of the scaffold, as indicated by the black arrows.

effective barrier to the transition state until it becomes surmountable by thermal energy. ${ }^{20}$

The force at which duplex DNA yields under tension depends on duplex length, ${ }^{19,21}$ base sequence, and any periodicity therein, ${ }^{22}$ force application geometry, whether static or varying tension is applied and for how long, ${ }^{18-20}$ and solution conditions including temperature ${ }^{19,23}$ and salt concentration. $^{24,25}$ Under shear stress, when tension is applied at either end of one strand of the duplex, DNA duplexes typically unbind at forces between 30 and $65 \mathrm{pN},{ }^{8,21}$ while in an unzipping geometry, where tension is applied to both strands at the same end of the duplex, yielding occurs at forces between 10 and $20 \mathrm{pN}^{18,26,27}$ The marked difference between the critical forces in shearing and unzipping geometries can be understood by considering the extension gained per base pair broken in each case. Since a force $F$ biases the unfolding landscape by an amount $\sim F z$, with $z$ the extension of the DNA along the force direction, the breaking of a single base pair in a shearing geometry, which allows an extension gain of $\Delta z / \mathrm{bp}$ $\sim 0.3 \mathrm{~nm}$, will be less favorable than base pair breakage in an unzipping geometry, which allows for an extension gain of $\Delta z$ / bp $\sim 1.3 \mathrm{~nm} .{ }^{19}$

The drive to produce DNA modules sufficiently rigid for use as building blocks for crystalline arrays and nanomechanical devices cultivated more complex arrangements of the wellstudied DNA duplex, ${ }^{28,29}$ namely, DNA helices linked by multiple crossover junctions. One of the simplest and earliest of these designs is two helices bundled together by a double crossover (DX) to form a modular tile. ${ }^{30}$ Such basic tile motifs can be expanded to construct 3D structures, including triangular tensegrity lattices, ${ }^{31}$ polyhedra, ${ }^{32}$ and nanotubes. ${ }^{33}$

The advent of DNA origami ${ }^{34}$ - a construction technique employing kilobase-long scaffold strands joined together by shorter, staple strands to form arbitrary shapes-allowed the construction of even larger and more intricate designs, which expanded the reach of DNA nanotechnology to nanosensing, ${ }^{35,36}$ nanomedicine, ${ }^{37}$ and nanoelectronics. ${ }^{38}$ Finally, a similar, but scaffold-free, approach was proposed by Yin and coworkers, ${ }^{39}$ in which only short single strands with four distinct binding domains are used. These versatile single-stranded tiles (SSTs) can also be fashioned into a wide array of $2 \mathrm{D}^{40}$ and $3 \mathrm{D}^{41}$ shapes.

As many applications depend on the increased structural rigidity of nanostructures as compared to duplex DNA, there has been a significant effort to characterize their elastic properties. Ligation closure experiments on the DX tile revealed a persistence length about twice that of duplex DNA. $^{42}$ Even larger persistence lengths, $\sim 10-20 \times$ that of duplex DNA, have been achieved with nanotubes comprised of six helices; ${ }^{43,44}$ for bundles of more helices, persistence lengths $40-200 \times$ that of dsDNA have been observed. ${ }^{45}$ Computational models have also contributed to the exploration of nanostructure mechanical behavior. At the finest levels of detail, atomistic MD simulations have successfully captured the structural rigidity of simple DNA origami and SST structures. ${ }^{46,47}$ Coarse-grained descriptions such as finite-element models ${ }^{48,49}$ and models that coarse grain at the level of multiple base pairs ${ }^{50}$ or single nucleotides ${ }^{51}$ have likewise provided useful analyses of the mechanical flexibility of origami structures.

More sophisticated applications of DNA nanotechnology require an understanding of the force response beyond that of the basic elastic properties. Such understanding is crucial particularly for applications that exploit behavior under force, such as biosensors, ${ }^{36,52,53}$ springs, ${ }^{54}$ force probes, ${ }^{55,56}$ and tensegrity structures. ${ }^{57,58}$ Further, characterizing the long-term stability of nanostructures under constant or intermittent stress is necessary for their use as movable joints and mechanical components like hinges ${ }^{59}$ and as hybrid nanopores, which undergo a constant denaturing stress from ionic current flow. ${ }^{60,61}$ The recent use of DNA nanotube hydrogels to study theoretical models of semiflexible polymers underscores the importance of knowledge of DNA nanostructural stability under force, as it hinged upon the robustness of the nanotubes under shear force. ${ }^{62}$

Given their substantial contribution to our understanding of the DNA duplex, force spectroscopy experiments are a logical stride in the development of DNA nanotechnology. Experiments have only just begun to explore the more complex force- 


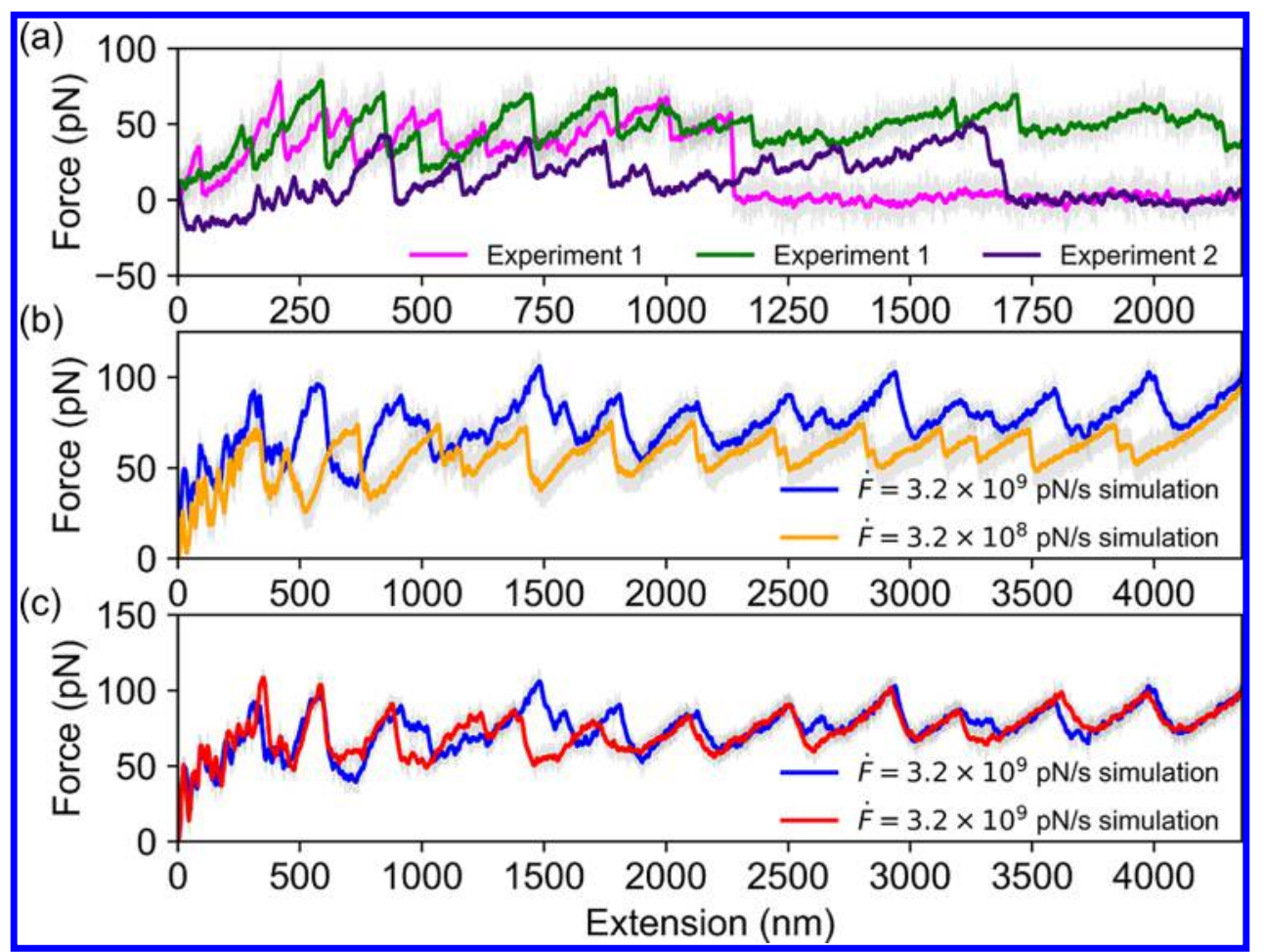

Figure 2. Force-extension curves for the Rothemund tile. (a) Experimental AFM curves at force loading rates of $\dot{F}=1.8 \times 10^{5} \mathrm{pN} / \mathrm{s}(\mathrm{green}$ and pink) and $\dot{F}=1.4 \times 10^{4} \mathrm{pN} / \mathrm{s}$ (purple). (b) Simulated pulling curves at $\dot{F}=3.2 \times 10^{9} \mathrm{pN} / \mathrm{s}$ (blue) and $\dot{F}=3.2 \times 10^{8} \mathrm{pN} / \mathrm{s}($ orange $) .(\mathrm{c})$ Simulated pulling curves, both collected at $\dot{F}=3.2 \times 10^{9} \mathrm{pN} / \mathrm{s}$; the blue curve is identical to the one shown in (b), reproduced here to facilitate comparison between pulling simulations carried out at the same loading rate. In all cases, colored traces are average curves and represent effective sampling rates of $250 \mathrm{~s}^{-1}$ (experiment), $1.3 \times 10^{7} \mathrm{~s}^{-1}$ (simulated, slower rate), and $6.6 \times 10^{7} \mathrm{~s}^{-1}$ (simulated, faster rate).

response behavior of DNA nanostructures, however; magnetic tweezer experiments have revealed torsional rigidities of origami 6-helix bundles in excess of $\sim 4 \times$ those of dsDNA, ${ }^{43}$ and recent optical tweezer experiments on origami nanotubes found disassembly forces of $40-50 \mathrm{pN} .^{63}$ Here, we build a more comprehensive picture of DNA origami force response by combining coarse-grained simulations with AFM experiments to characterize the unraveling of a standard origami system: the Rothemund tile. $^{34}$ We also perform simulations of two previously published experimental systems for which forceextension data are available: a 10-helix bundle $\mathrm{e}^{64}$ and a biosensor $^{36}$ designed such that its force-extension behavior signals the presence of aptamers in solution.

By pursuing a combined simulation and experimental approach, we are able to correlate features in force-extension data directly with underlying structural changes, yielding insights inaccessible via experiment alone. Finite-element approaches to modeling origami and models that coarse grain on scales larger than a single nucleotide cannot capture the base-pair breakage fundamental to force spectroscopy studies. ${ }^{49}$ On the other hand, while capable of investigating unravelling, all-atom origami simulations cannot currently probe time scales longer than $\sim 100 \mathrm{~ns}$ or structures larger than a few thousand base pairs. ${ }^{46}$ To access large origami systems on time scales relevant to experiments while maintaining the ability to describe base pair breakage, we therefore use oxDNA, ${ }^{65,66}$ a nucleotide-level coarse-grained model. Because of oxDNA's previous success in reproducing DNA nanostructures, ${ }^{51,67,68}$ capturing DNA mechanical response to tension, ${ }^{19,69}$ and describing DNA twist, ${ }^{70,71}$ it is well-suited to the present study.

By forcibly unravelling DNA origami, we explore departures from duplex behavior and probe the basic physics of origami force compliance, as well as the dynamics of force propagation through the structures. We also probe the mechanical limits of DNA nanostructures under external stress, knowledge of which is crucial for applications that utilize nanostructures to apply or resist force. ${ }^{54,57,58}$ Our results provide further validation of oxDNA's robustness in treating DNA origami. More importantly, they illustrate the possible fruits of combining experimental force spectroscopy with simulation to explore unfolding pathways and examine origami mechanical resistance to force in extensive detail, which can inform rational design of future nanomechanical structures.

\section{RESULTS AND DISCUSSION}

Rothemund Tile. Our system, shown in Figure 1a,b, is very similar to Rothemund's original origami design, although we retain a 20-bp stem hairpin that Rothemund excised. The 6909nucleotide scaffold is bound by 30-nucleotide (nt) and 32-nt staples, most of which have three domains, with one long (binding) and two short (linker) domains. The design has 24 rows with a central seam dividing the origami into two equivalent halves, and a very regular pattern of staples (Figure S1).

To mimic the AFM-pulling procedure, in which one end of the enzymatically linearized scaffold is fixed to a glass coverslip via maleimide-thiol coupling and the other to the retractable AFM cantilever via noncovalent biotin-streptativin link, simulations were performed by placing the ends of the tile in harmonic traps (see arrows in Figure 1b) and moving one relative to the other at a constant rate. Simulated force loading rates were $3.2 \times 10^{8} \mathrm{pN} / \mathrm{s}$ and $3.2 \times 10^{9} \mathrm{pN} / \mathrm{s}$, and experimental force loading rates were $1.4 \times 10^{4}$ and $1.8 \times$ $10^{5} \mathrm{pN} / \mathrm{s}$. By "force loading rate", we mean the average rate of 

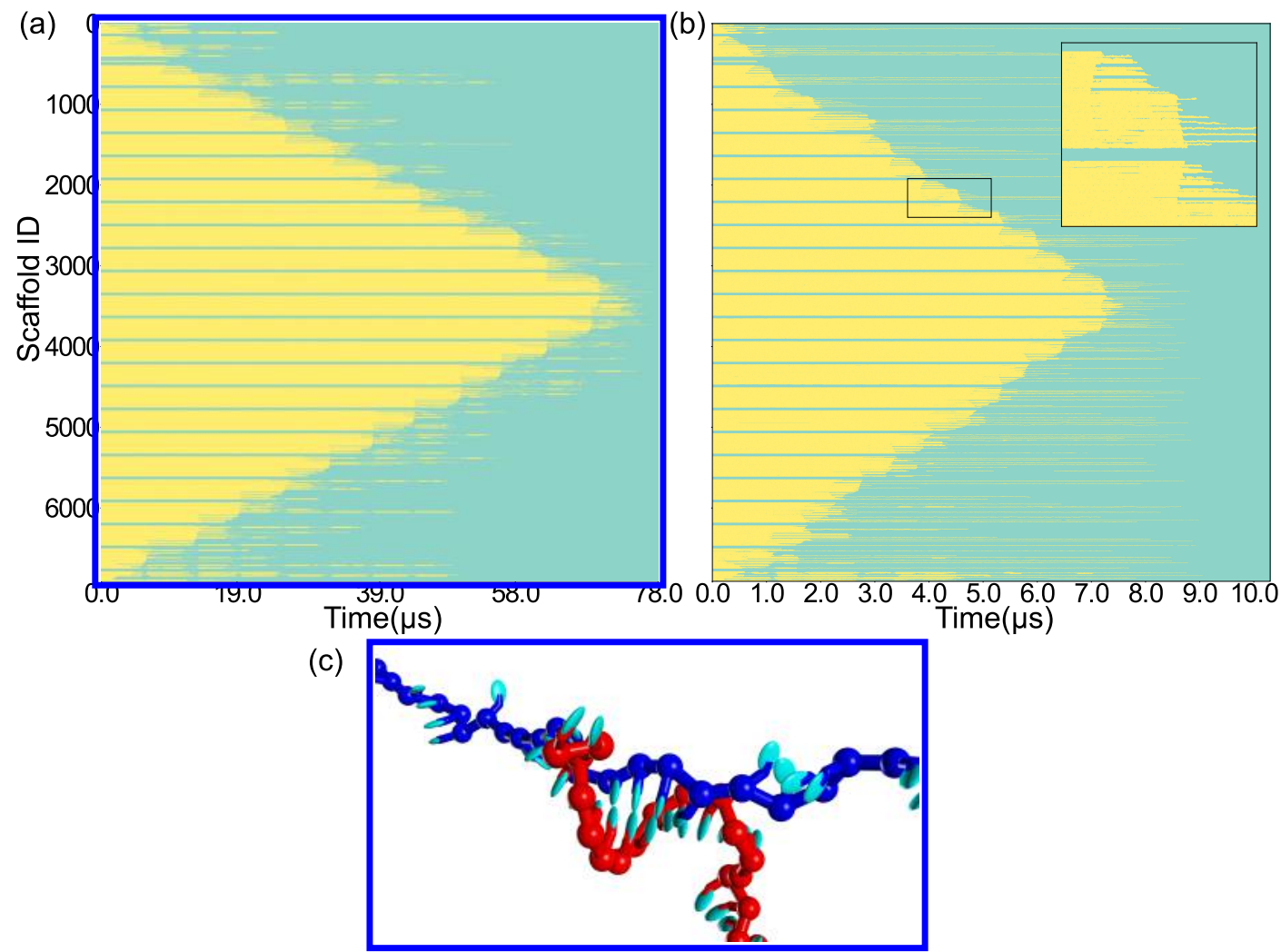

Figure 3. Pattern of native-bond unfolding in pulling simulations of the Rothemund tile. Yellow regions indicate bonds that are still in their native configuration; blue regions indicate an unbonded scaffold nucleotide. (a) Slower pulling rate; (b) faster pulling rate, corresponding to the blue trace in Figure $2 \mathbf{b}$,c. Inset: detail of the unfolding mechanism for three half rows. Nucleotide 0 corresponds to the end associated with the moving trap. (c) Illustration of a "persistent" staple domain (red) with three remaining base pairs connecting it to the scaffold (blue); these last base pairs do not prevent the scaffold backbone aligning with the force, which makes them more resistant to force-induced melting. By "persistent domain", we mean a domain that remains bound after the part of the scaffold it is on has been pulled away from the main structure; such domains are visible as the thin yellow lines in (a) and (b).

force increase as given by the product of harmonic trap stiffness and speed. This value is thus an instrumental property, and the loading rate the molecule actually "feels" differs due to the presence of linker strands as well as the particular configuration of the molecule; estimates of the experimental force loading rate from fits to force-extension curves yield values $1-3$ orders of magnitude lower than instrumental loading rates. While our simulated loading rates are many times greater than the experimental loading rates, any conversions of coarse-grained time scales to absolute time units must be interpreted with caution. The values above give a "worst-case" disparity; since coarse-graining speeds up diffusive dynamics relative to the microscopic time scales that set the coarse-grained time unit, ${ }^{72}$ the resulting telescoping of time scales ${ }^{73}$ renders the effective force loading rates for simulations closer to experiment than they would appear from the above values.

Figure 2 contains the experimental (a) and simulated (b and c) force-extension curves (FECs) for the Rothemund tile. The experimental curves terminate before the full structure is unraveled-which simulations indicate would occur around $4000 \mathrm{~nm}$-because the pulling handle between the molecule and the AFM tip breaks. For domains under mechanical load, there is a non-zero probability that statistically stronger ones break before weaker ones. This probability scales with the magnitude of overlap between the rupture force distributions of both domains. ${ }^{74,75}$ In the single-molecule force spectroscopy (SMFS) experiments performed here, the pulling handle is loaded throughout the whole retraction and force rarely drops to near zero between rupture events. The state of the pulling handle therefore does not get reset completely, and the probability of rupture increases with extension. It is thus unsurprising to see SMFS curves that do not unfold the whole origami structure in a single pulling cycle.

Nonetheless, several salient features are visible in the data. Immediately striking is the regular, sawtooth pattern present in all of the traces, a signature of the regularity of the tile design. The tile unravels in units evidenced by these sawtooth rips, indicative of cooperative unfolding within each subunit. Notable, though, is that each "rip" occurs at more or less the same rupture force: the unfolding of one unit does not render subsequent units easier to unfold.

Also evident in both experimental and simulated curves is the fundamental stochasticity of the process: even under an identical external protocol, the tile unfolds along a slightly different pathway every time. The pink and green experimental curves in (a), both collected at the same force loading rate, and the simulated curves in (c), also collected at the same rate, differ nonetheless from one another since barrier crossing is a thermally activated diffusive process. $^{76}$

Both the experimental and simulated FECs exhibit a decrease in the slope of the force rise preceding each sawtooth "rip" as more of the tile unravels, indicating a decrease in the apparent force loading rate. ${ }^{77,78}$ This is a direct consequence of the effective increase in the linker length as the origami subunits closest to the handles unfold, releasing ssDNA, which behaves as an entropic spring that couples to the harmonic traps to 
govern the overall force response of the system. The elastic behavior of ssDNA-typically modeled as a modified freely jointed chain ${ }^{1}$ or a worm-like chain ${ }^{79}$-is highly nonlinear and is known to reduce the loading rate transmitted to a molecule. ${ }^{77}$ Thus, as more ssDNA unravels, the apparent force loading rate decreases. ${ }^{77}$ This accounts for the sharper, more jagged features visible near the beginning of the traces being softened, as sharp features are smoothed through convolution with the soft force response of the increasingly long polymeric tethers. ${ }^{80}$ The stochasticity in the force-extension traces is more pronounced the farther from equilibrium the protocol is; in the (nonphysical, $\dot{F}=0$ ) limit of quasi-static pulling, the traces would appear identical for every realization. As the apparent force loading rate decreases, therefore, so too does the variation from pull to pull. We can rationalize this by considering that the width of the characteristic rupture force distribution for a given structural element increases with force loading rate, so a smaller spread in observed rupture forces is expected as loading rate decreases. ${ }^{81,82}$ This explains the increased regularity and reproducibility of features as the extension grows, apparent in Figure $2 \mathrm{c}$. We note that the final segment of the simulated force-extension curves corresponds to the force response of ssDNA.

The FECs also exhibit a dependence of rupture force on probe velocity, with the force required to unravel specific features increasing with pulling speed. This behavior of rupture force under dynamic loads was first described by Evans and Ritchie, ${ }^{20}$ following Bell, ${ }^{83}$ who predicted that rupture force is an increasing function of pulling speed. Later, more refined theories have corroborated this, ${ }^{81,82,84}$ and experiments are frequently analyzed using this framework. ${ }^{5,85,86}$ In this context, we can rationalize the maximum rupture force increasing from $\sim 50 \mathrm{pN}$ for the lower experimental loading rate to $\sim 75 \mathrm{pN}$ for the higher experimental loading rate, and from $\sim 65 \mathrm{pN}$ for the slower simulated rate to $\sim 100 \mathrm{pN}$ for the fastest simulated rate. We reiterate that comparing simulation and experimental time scales is a subtle task, as coarse graining speeds up intrinsic dynamics. $^{72}$

A key advantage of simulations lies in the ability to correlate force-extension data with structural changes. Figure 3 tracks the breakage of native base pairs as the tile unravels for two pulling speeds. By "native", we mean staple-scaffold base pairs designed to be present in the correctly folded tile. At $t=0$, the tile is fully natively bonded. Each yellow block corresponds to two half-rows: the blue "stripes" punctuating the yellow regions are regions of single-stranded scaffold, which can be seen in Figure $1 \mathrm{~b}$ at the end of each row. The nucleotide labeled " 0 " is subjected to the moving harmonic trap, while the nucleotide labeled "6908" sits in a stationary trap. Clearly, the structure unravels from the scaffold ends inward, corresponding to the location of applied force.

From the plots of base pair breakage versus time, we can glean the basic mechanism by which the Rothemund tile unfolds. First, note that the routing pattern for the tile's rows means that the force is alternately antiparallel and parallel to the duplex; see Figure 4. The gain in free energy per broken base pair is $\sim \Delta z / \mathrm{bp}$, where $\Delta z / \mathrm{bp}$ is the extension gain per base pair broken. For rows where the scaffold strand is antiparallel to the force (Figure 4a), each broken base pair contributes the base-base distance along the backbone plus the rise of a base pair in the duplex, in oxDNA 9.8 $\AA$. By contrast, when the scaffold strand is parallel to the force (Figure $4 \mathrm{~b}$ ) $\Delta z / \mathrm{bp}$ is a more modest $\sim 3 \AA$, the difference between the backbone

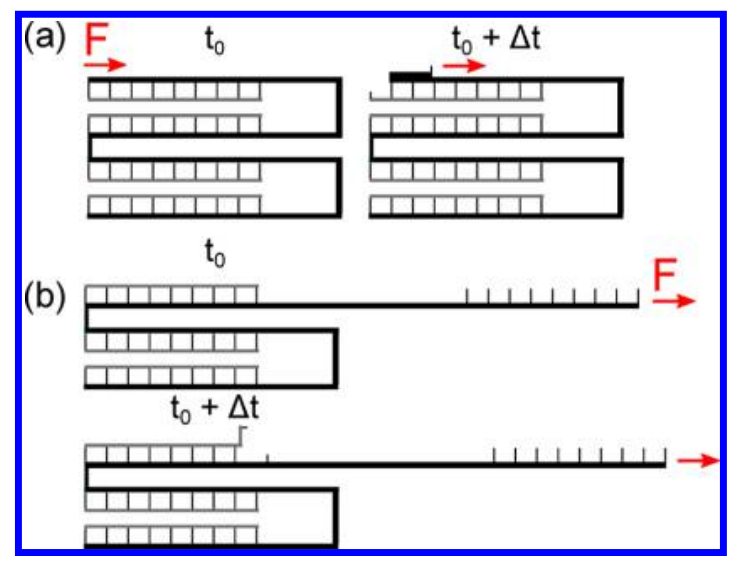

Figure 4. Antiparallel (a) and parallel (b) force application modalities. When force is applied antiparallel to the helix, the geometry is "unzipping-like" and carries an extension gain per broken base pair of $9.8 \AA$. Applying force parallel to the helix, or in a "shear-like" geometry, by contrast only yields an extension gain per broken base pair of $\sim 3 \AA$. This difference renders the forces observed for rows that are "sheared" much higher than those for rows that are "unzipped".

length and the base-pair rise of the duplex. Consequently, the force required to break a base pair in the parallel geometry is much greater than the force required to break a base pair in the antiparallel geometry. This is analogous to the fact that the DNA duplex rupture force for a shear geometry is much higher than that for an unzipping geometry.

Consider the upper half of Figure 3a,b. The top half of each yellow block corresponds to a half-row with "shear-like", or parallel, pulling geometry, and the bottom half corresponds to a half-row with "unzipping-like", or antiparallel, pulling geometry. The inset of Figure $3 \mathrm{~b}$ shows the unfolding of three half-rows: in parallel pulling geometry (upper half of top yellow block), antiparallel pulling geometry (lower half of top yellow block), and again in parallel pulling geometry (upper half of bottom yellow block).

The force increases roughly linearly in the parallel pulling geometry until a maximum force is reached that allows a full half-row to be sheared off. Once the final seam staple in this half-row is removed, the subsequent half-row, now in antiparallel pulling geometry, can begin to unfold immediately because the force already exceeds the critical unzipping force. Most of the base pairs in the lower half of each yellow blockthe ones that are "unzipped"- break over a short period of time, as evident in the inset of Figure $3 \mathrm{~b}$. The consequent rapid release of scaffold strand leads to the sudden drop in the force seen in the sawtooth features of Figure 2. Also, for those staples whose long domain is in the half-row that unzips, yielding generally occurs by the unbinding of the shorter domain from the half-row below it, leaving the staple attached to the released scaffold. This is visible in Figure 3 as a series of blue lines in the top halves of the yellow blocks (the half-rows in "shearing" geometry), as well as the thin yellow lines associated with "persistent domains" in the bottom halves of the yellow blocks (corresponding to "unzipping" geometry). By "persistent domains", we mean regions where staples remain partially bound by a single domain to the scaffold strand even after the part of the scaffold they are on has been pulled away from the main structure.

This mechanism, wherein two rows unravel in parallel, leads to the regular features in the FECs. This can be quantified by 


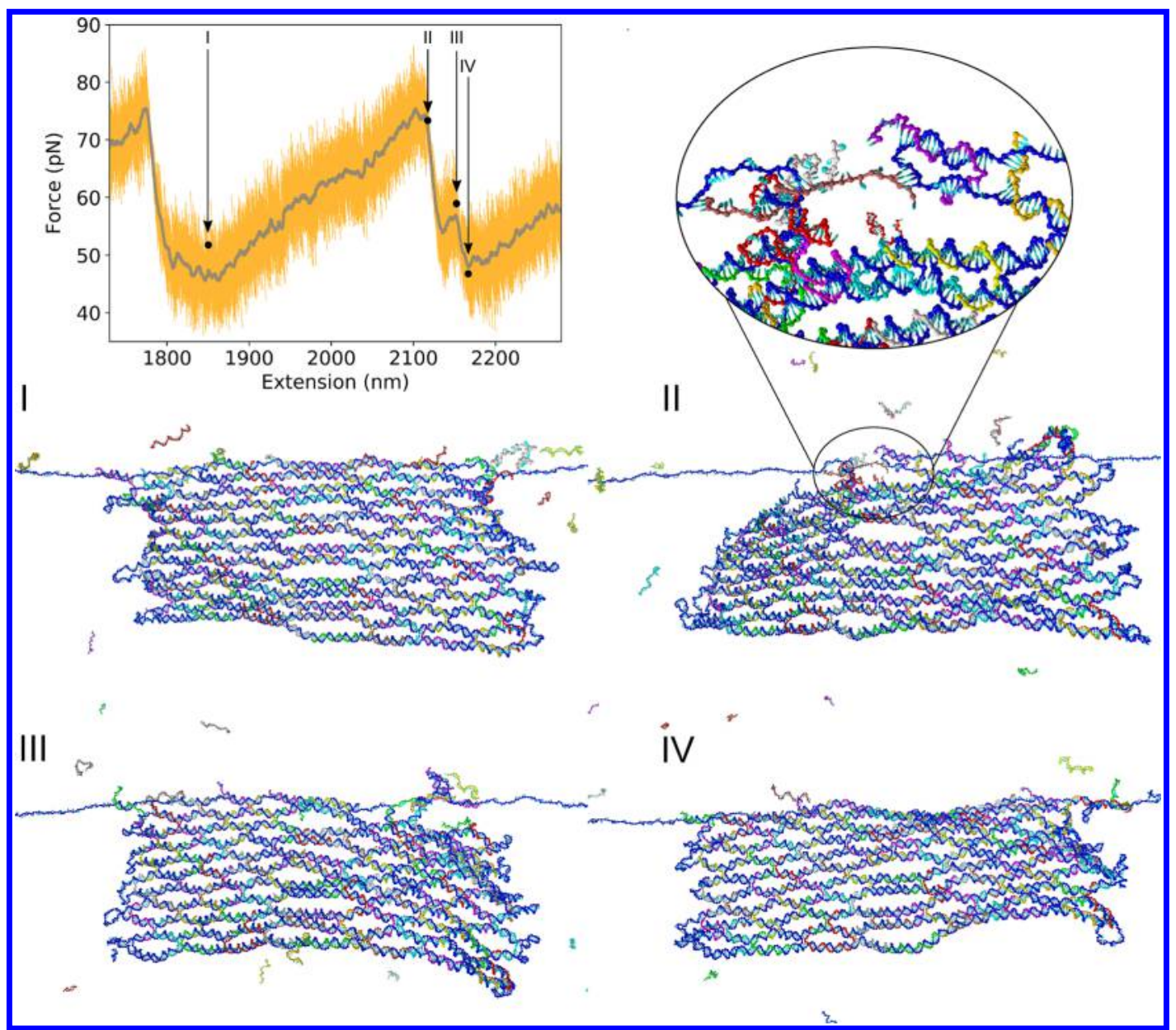

Figure 5. Structural illustration of the unfolding mechanism of the tile. A section of a simulated force-extension curve at $\dot{F}=3.2 \times 10^{8} \mathrm{pN} / \mathrm{s}$ is shown; the gray curve is an average. I-IV are configurations at different times, as marked on the force-extension curve. I denotes a configuration at the beginning of a shearing mode. The sawtooth feature that peaks at point II corresponds to the top row being sheared open (see also blowup); subsequently, the next row unzips. The small bump at III occurs because the right half of the row unzips at a slightly later time than the left. Like I, IV is a configuration at the beginning of a shearing mode, but after two rows have been pulled off.

performing a Lomb-Scargle ${ }^{88}$ periodicity analysis of one of the FECs in Figure 2. Figure S4 shows a strong peak that occurs very close to the length of two rows of the tile.

An additional feature evident from Figure 3 is that there is a tendency for the corresponding left-hand and right-hand pairs of half-rows to yield almost simultaneously, giving these plots their rough mirror symmetry. This tendency is more evident both at the slower pulling rate, and at later times. One of the effects of this synchronization is to reduce the variability in the FECs at later times.

Some asymmetry in the application of force is visible in the plots: persistent domains are more numerous in the lower half of the plots, corresponding to the half of the structure furthest from the moving trap. For example, in the faster simulations, at $5.0 \mu \mathrm{s}$ in Figure $3 \mathrm{~b}$ (after 15 rows have unfolded), there are 39 persistent domains in this lower half, while the upper half contains only 22 such domains. Similarly, at $8.0 \mu \mathrm{s}$, after all rows have unfolded, 44 persistent domains remain in the lower half and only 21 remain in the upper region. This asymmetry is an indication that the time scale over which force is increasing is faster than the time required for the force to propagate through the structure; that is, one-half of the tile "feels" a somewhat larger instantaneous force than the other half, making the force-induced melting of these persistent domains more likely. This effect is thus more pronounced for the faster pulling rate. Indeed, in the slower simulations, there is much less asymmetry in the persistent domains; e.g., just after $\sim 34.0$ $\mu$ s (after 11 rows have unfolded), there are 10 persistent domains in the lower half and 13 in the upper half. Such delayed propagation of stress along polymer chains has been noted elsewhere. ${ }^{89}$

A further difference between the two rates is visible in the recurrent breakage and reformation of base pairs in persistent staple domains, which occurs much more frequently at the slowest pulling rate. When the force approaches a maximum, the persistent domains begin to melt and the number of base pairs in these domains diminishes; but once the "rip" has occurred and the force in the system decreases, at the slowest rates there is sufficient time for the domain to reanneal. Note that the last few base pairs between staple and scaffold are significantly more resistant to force-induced melting, because the scaffold backbone can then approximately align itself with the direction of the force and so the extensional gain from strand melting is much reduced; ${ }^{19}$ see Figure $3 c$.

The simulations also allow us to directly visualize the changes in configuration underlying these events. Figure 5 depicts the mechanisms underlying a typical "sawtooth" feature in the FEC. At point $\mathrm{I}$, the top row is in a "shear-like" pulling geometry. As 
force increases, the base pairs become increasingly strained, particularly on the left-hand side of the tile, corresponding to the moving harmonic trap. Structure II reveals the tile immediately after the final staple of the topmost left-hand half-row-the central seam-has been broken, but with the seam staple between the lower two half-rows still just in place. The half-row underneath then begins to unzip, and the lefthand half-row has completely unzipped by stage III. The consequent removal of the second bridging seam staple has a significant effect on the unravelling of the right-hand side. It allows the section nearest the seam to rotate about the nearest junction, dramatically changing the geometry of the pulling. At III, unzipping of the second half-row on the right-hand side has begun, even though the staples nearest the seam are still intact in a subunit that is now roughly vertical. The small peak in the FEC at III corresponds to this yielding of the right-hand side. The key role of the seam staples underlies the synchronized unravelling of the pairs of half-rows. Two rows, corresponding to the basic sawtooth unit in the force-extension plots, have been unravelled between I and IV-further confirmation of our picture of the basic mechanism of unfolding for the Rothemund tile.

Ten-Helix Bundle. The 10-helix bundle was originally designed by Bae et al. ${ }^{64}$ to explore the concept of mechanical origami assembly as an alternative to annealing-the scaffold was held at constant tension to eliminate transient secondary structure as folding progressed-but the force-extension curve associated with disassembly was also probed. It is both topologically and structurally more complex than the Rothemund tile. First, the staple routings on the 1768nucleotide scaffold are more nonlocal, with some staples spanning up to 5 domains (see Figure S2). Second, the structure is $3 \mathrm{D}$ rather than $2 \mathrm{D}$. Despite these differences, the simulated FECs share many common features with those of the Rothemund tile: the basic sawtooth-like, "stick-slip" dynamics, shown in Figure 6; an overall increase in rupture forces as pulling rate increases; and increased reproducibility with increasing extension in the traces performed under the same conditions.

One consequence of the more complex structure, however, is that there is a less obvious preferred folding pathway; a

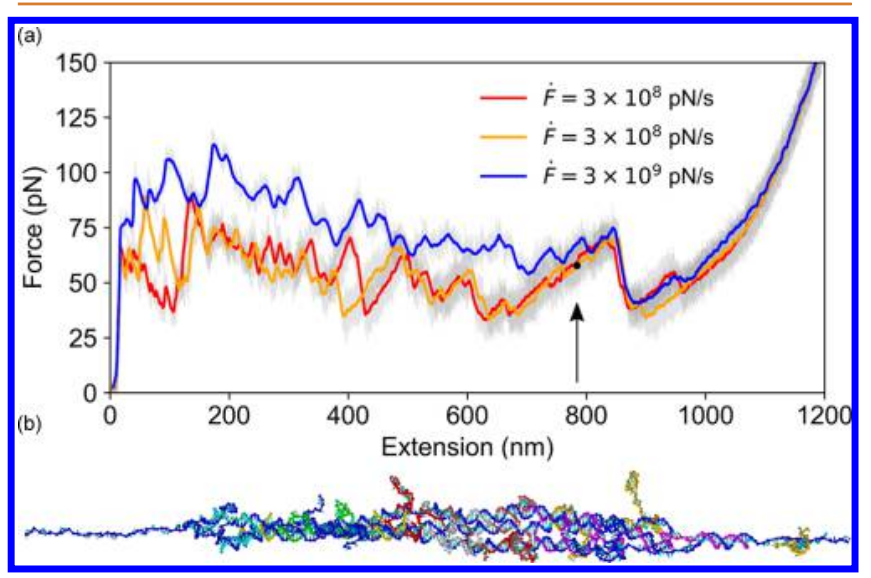

Figure 6. (a) Force-extension curves corresponding to unfolding the 10-helix bundle with moving harmonic traps at loading rates of $\dot{F}=3.2 \times 10^{9} \mathrm{pN} / \mathrm{s}$ (blue) and $\dot{F}=3.2 \times 10^{8} \mathrm{pN} / \mathrm{s}$ (red and orange). (b) Structural snapshot, corresponding to the black marker and arrow in (a), of the final rupture feature in the forceextension curves: three helices are in a shear geometry. multiplicity of possible unfolding events may yield the same change in extension. Figure 7 shows that the helix bundle samples different unfolding pathways at different loading rates, and Figure 8 establishes that multiple unfolding pathways are sampled even under identical simulation conditions. For example, in one simulation, the bundle unfolds along a single helix before other helices unravel (Figure 8, right panel). In another trace, collected under identical simulation conditions, other helices begin to yield from the opposite scaffold end before the first is finished unravelling (Figure 8, left panel). Even along a single pathway, there is no clear uniformity in terms of a repeating unfolding unit; Figure S4 reveals an absence of periodic signatures in Figure 6a associated with any particular length scales.

Also notable is the fact that in many regions, successive rupture events occur at progressively lower forces. For all traces, the highest forces reached occur near the start of the unravelling process. This "strain-softening" effect arises because, unlike with the Rothemund tile, the gain in extension per base pair broken varies significantly as the helix bundle unravels. Figure 9 reveals the difference: whereas the behavior of $\Delta z / \mathrm{bp}$ is essentially linear for the tile, the helix bundle $\Delta z /$ bp contains regions of curvature, wherein breaking of key domains renders the structure increasingly compliant; it "catastrophically" fails. This unfolding behavior has been described previously as "shielded" unfolding, in which structural topology prevents weaker barriers from being compromised before stronger ones. ${ }^{90}$ A specific structural example of this shielding is shown in Figure 10: once the first full helix has unravelled at $\sim 170 \mathrm{~nm}$, the remainder of the bundle is comparatively easier to unfold. Given the multidomain nature of the staples, unravelling the first helix weakens many of the staples holding subsequent helices in place; this gives rise to the strain-softening observed. The plots in Figure 7 (particularly b) illustrate the weakening of the interior regions of the bundle. Unravelling does not simply occur from the ends inward, as with the tile; as the final failure is approached, the structure appears to separate into multiple blocks. Strain-softening is visible up to $\sim 650 \mathrm{~nm}$ extension. At this point, as illustrated in Figure $6 \mathrm{~b}$, three helices remain and force application occurs roughly in a shearing geometry, accounting for the $\sim 75 \mathrm{pN}$ force required to unfold this final feature-not far below the initial maximum force of $\sim 85 \mathrm{pN}$.

Employing a linearly increasing force protocol rather than harmonic traps makes the bundle's catastrophic failure even more evident: Figure 11 contains FECs for a range of force loading rates, where scaffold ends are both held at the same, linearly increasing, tension. Again, we note the increase in rupture force for increasing force loading rate. After a few initial rupture events, visible in the inset of Figure 11, the bundle essentially unfolds 'all at once,' evidenced by the long linear regions in the force-extension data. Once the monotonically increasing force surpasses the critical force at which all landscape barriers are removed, unfolding enters a purely downhill regime. Thus, the force application protocol is clearly of central importance in the analysis of molecular mechanical properties; in the case of harmonic traps, the molecule has opportunities to partially relax, allowing us to distinguish more detailed features in the force-extension curves.

Bae et al. ${ }^{64}$ also noted cooperative unfolding of the helix bundle, which in their magnetic tweezer pulling experiments began to yield at $\sim 30 \mathrm{pN}$, a rupture force half as large as for our systems, which yield at $\sim 60 \mathrm{pN}$ under the slowest pulling rate. Their experimental force loading rate was not provided, but it 

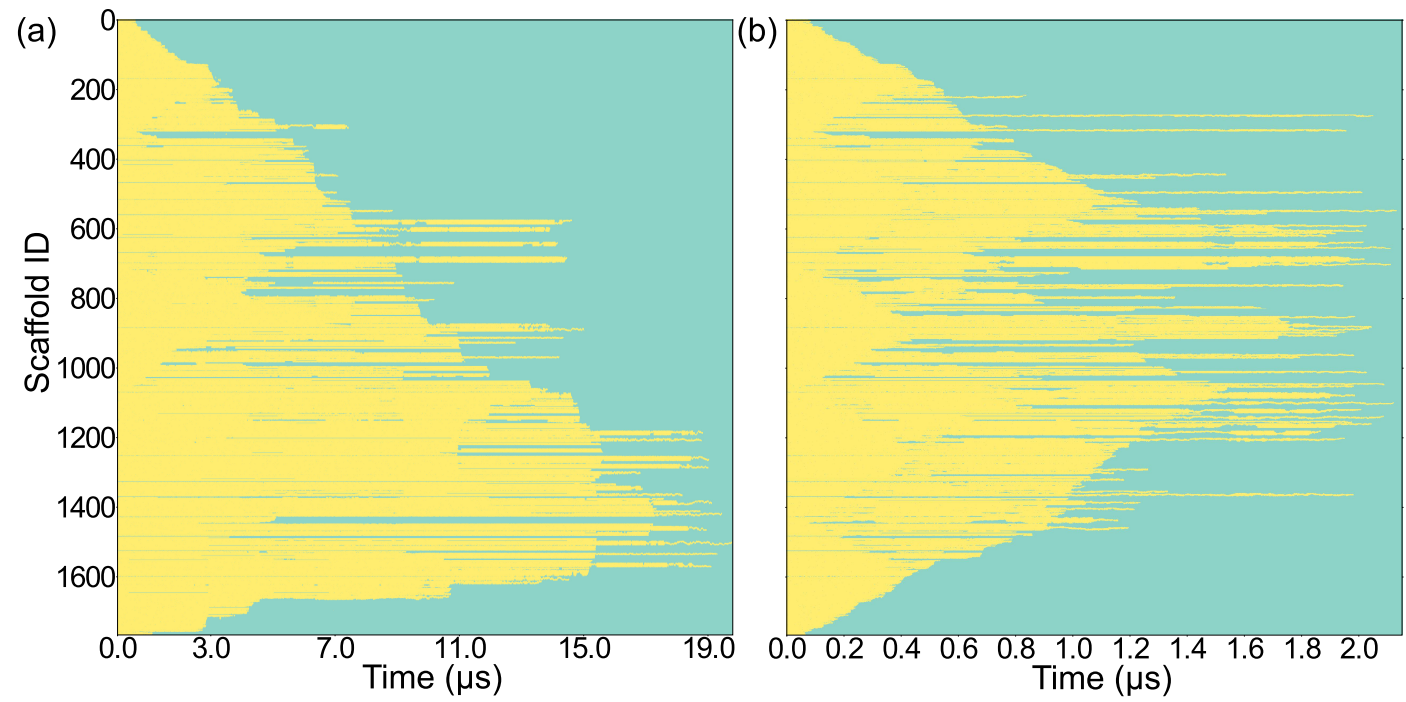

Figure 7. Pattern of native-bond unfolding in pulling simulations of the 10 -helix bundle at $(\mathrm{a}) \dot{\mathrm{F}}=3.2 \times 10^{8} \mathrm{pN} / \mathrm{s}$ (the dark red curve in Figure $6 \mathrm{a}$ ) and (b) $\dot{F}=3.2 \times 10^{9} \mathrm{pN} / \mathrm{s}$ (the blue curve in Figure 6a). Yellow regions indicate bonds that are still in their native configuration; blue regions indicate an unbonded scaffold nucleotide. In contrast to the Rothemund tile, the plot for the faster pulling rate is more symmetric because the faster rate makes it less likely for the structure to find the more favorable asymmetric unfolding pathway.

was certainly smaller than our slowest rate, which probably accounts for most of the discrepancy.

It is noticeable that there is a pronounced asymmetry with respect to scaffold ends in Figure 7a, but to a much lesser extent for the faster pulling rate, Figure $7 \mathrm{~b}$. This pulling-rate dependence is the opposite of the behavior observed for the tile (Figure 3a,b) and is a result of an asymmetry in the helix bundle's design. Specifically, there is an additional nick close to the end of helix 3 (see Figure S2) that will lead to a somewhat lower barrier for unravelling to be initiated from this end. If initiation occurs from just one end, the origami will then tend to rotate so that the points at which the force acts on the origami are colinear, which in turn leads to a greater extensional release if that row continues to unravel. Furthermore, the resulting localized strain-softening will make it more likely that subsequent unraveling will continue from this end. When the pulling rate is higher, as in Figure $7 \mathrm{~b}$, the system has less time to find the most favorable pathway, and a more symmetric picture emerges.

Seven-Tile Biosensor. To illustrate the insights that can be obtained even for very large systems, for which simulated pulling rates are necessarily much greater than experimental rates, we also simulated the seven-tile biosensor of Koirala et al. ${ }^{36} 14$ 761-nucleotide origami consists of seven rectangular tiles joined by 44-base pair duplex "locks", where the breaking of the locks can be observed as a large change in extension due to the connected tiles then being able to reorient along the direction of applied force. In the experiments, the locks opened sequentially. Even at our slowest pulling rate of $\dot{F}=3.2 \times 10^{8}$ $\mathrm{pN} / \mathrm{s}$, we observed the locks opening at the same time (Figures S9 and S10). Nevertheless, the locks break in the order expected, outermost to innermost; Figure 12 illustrates the unlocking mechanism. Despite the fact that the resolution of individual events is not comparable to the experimental forceextension curves ${ }^{36}$ given the relatively high force loading rate, the signature of the locks opening can still be seen in the force-extension data (Figure S10). Furthermore, at this high rate, the tiles themselves begin to unravel somewhat before all of the locks have broken, a sign that our simulations are far from equilibrium. This example of a large system illustrates some of the limits of of our simulation method; however, we are still able to capture salient insights, indicating that simulated SMFS can still be valuable for very large systems.

\section{CONCLUSION}

We have used molecular simulations performed using the oxDNA coarse-grained model to characterize the mechanical force response of two archetypal DNA origami systems, the Rothemund tile and a 10-helix bundle, and to explore the force response of a seven-tile biosensor. We also performed AFM pulling experiments on the Rothemund tile, enabling us to correlate features in experimental data with structural changes revealed in simulation. Both experiments and simulations exhibited regular, sawtooth unfolding behavior in the forceextension curves for the Rothemund tile and captured yielding at similar forces, $\sim 65 \mathrm{pN}-75 \mathrm{pN}$.

Our force-extension data yield insight into the complex freeenergy landscapes for origami unfolding, underscoring the much richer force-induced melting behavior of origami compared to dsDNA. We observe stochasticity in the unfolding process for both systems; multiple pathways, cooperative unfolding, and strain-softening in the unfolding of the 10helix bundle; and geometry-dependent $\Delta z / \mathrm{bp}$. Since force biases the pathways taken through the unfolding landscape, we expect the assembly landscapes for these systems to exhibit even greater complexity.

Variations in rupture forces with pulling rates imply that any designed structural resistance to force will be loading rate dependent. ${ }^{19}$ The effect of design on force-response behavior has been highlighted by the disparate results obtained for the Rothemund tile, which unfolds modularly, and the 10-helix bundle, whose nonlocal staple routings lead to strain-softening and catastrophic failure. Differences between results obtained using linear and harmonic force pulling also underscore the importance of carefully planning the force application protocol in SMFS studies.

Our results also reveal a glimpse of the complexities of force propagation through origami structures, manifesting in 


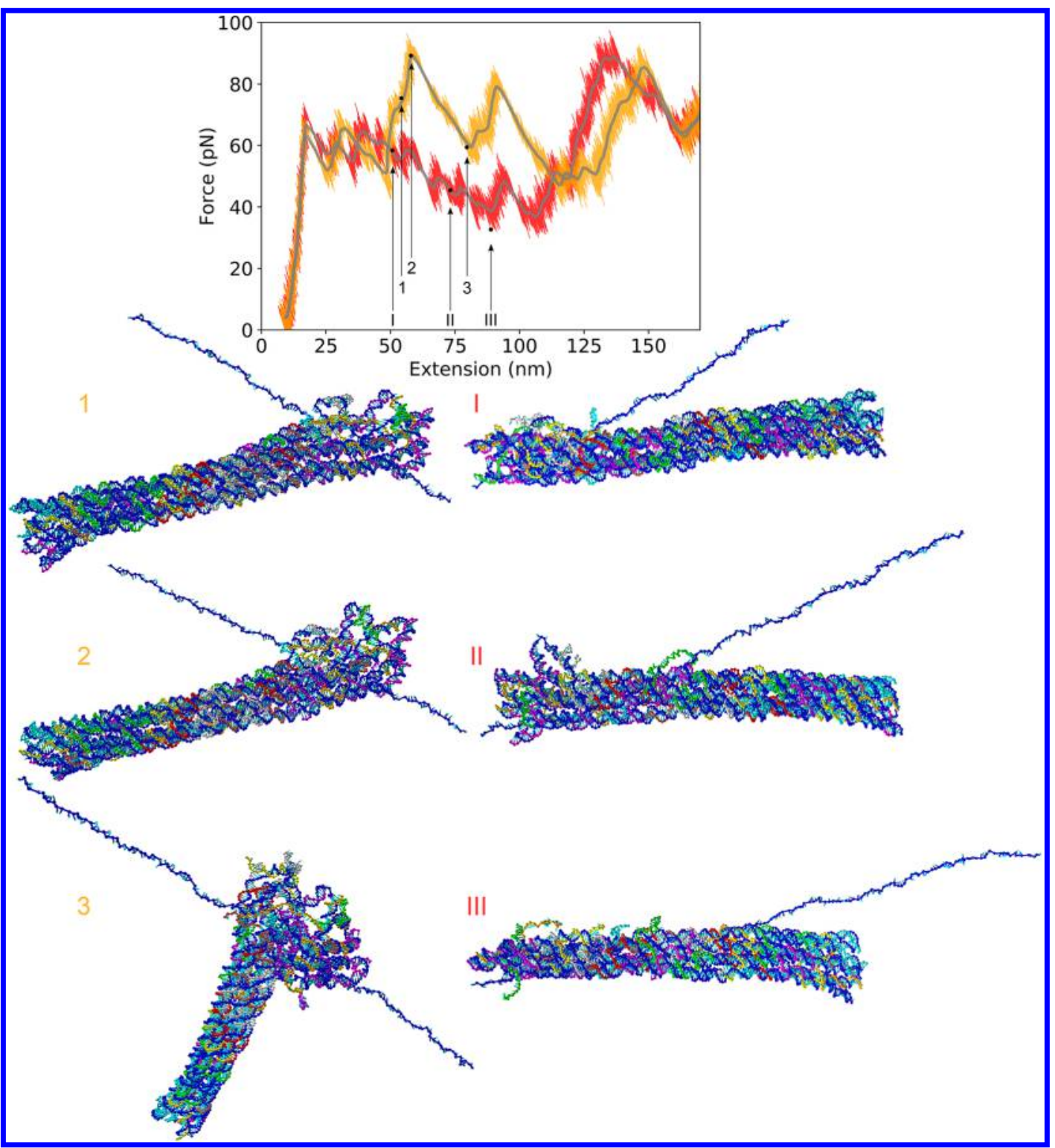

Figure 8. Structures exemplary of the stochasticity in the force-extension unfolding curves for the 10-helix bundle. Curves for each simulation, performed at the force loading rate $\dot{F}=3.2 \times 10^{8} \mathrm{pN} / \mathrm{s}$, are shown at top, and the locations of the structural snapshots are indicated. The unfolding pathway of the orange force-extension curve, shown on the left, exhibits unravelling from both ends before the first helix has completely yielded. The unfolding pathway of the red force-extension curve, however, features a complete unfolding of the first helix before others begin to yield.

asymmetric unravelling behavior. Valuable future investigations could consider in more detail how force propagation dynamics are complicated by the presence of junctions and multidomain staples.

We have demonstrated the possibilities for improving comprehension of origami mechanical response and informing rational design of nanostructures through joint simulation and experimental studies. Because it is capable of capturing the unravelling of large DNA nanostructures, our simulation strategy complements existing approaches to modeling the mechanical properties of origami, and we hope future work will continue to profit from the insights offered by DNA origami force spectroscopy.

\section{METHODS}

Experiments. Briefly, scaffold " 7560 " 91 from bacteriophage M13mp $18^{34}$ was double functionalized by ligation with biotin and thiol groups to facilitate attachment to either the streptavidin-coated AFM tips or maleimide-functionalized substrate, respectively. The circular, single-stranded scaffold was digested by BamHI-HF and EcoRI-HF after partial prehybridization at restriction sites. The linearized scaffold was purified by solid-phase extraction. Afterward, partially double-stranded scaffold was ligated with $10 \times$ excess of double-stranded inserts carrying either biotin or thiol ( $5^{\prime}$-BIO; $3^{\prime}$ THIOL $+5^{\prime}$-phosphate) by T4 DNA ligase. This ligated scaffold was either used in its unpurified form (Figure 2a, green and pink curves) to fold DNA origami rectangles ${ }^{92}$ or was purified from excess functionalization strands by size exclusion (Figure 2a, purple curve) in spin filtration columns and subsequently folded. The folding procedure has been reported elsewhere. ${ }^{92}$ The freshly folded DNA origami was purified by PEG precipitation. ${ }^{93}$ Prior to "unzipping" experiments, the origami pellet was resolved in $1 \times \mathrm{TE}$ buffer $(\mathrm{pH} 8)$ supplemented with $10 \mathrm{mM} \mathrm{MgCl} 2$ and $2 \mathrm{mM}$ TCEP. TCEP is required to reduce oxidized thiol-groups and enable maleimide conjugation reaction on surfaces. Afterward, the resolved origami samples were coupled to AFM sample slides. 


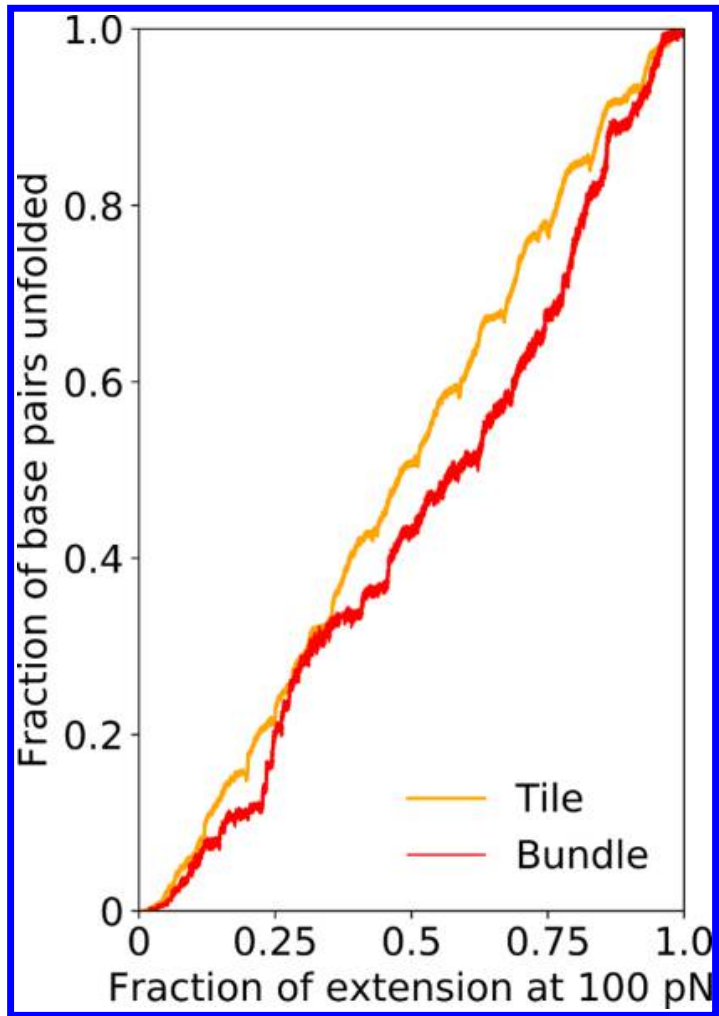

Figure 9. Fraction of native base pairs unfolded as a function of the fractional extension (measured with respect to the extension at 100 pN) for the Rothemund tile and 10-helix bundle for a pulling rate of $\dot{F}=3.2 \times 10^{8} \mathrm{pN} / \mathrm{s}$. The Rothemund tile exhibits a regular, linear pattern of $\Delta z / \mathrm{bp}$ as rows alternately shear and unzip. In contrast, the curve for the helix bundle is much more nonlinear, and curvature in the plot indicates a strain-softening, or shielding, effect: once certain key base pairs unfold, subsequent base pairs are easier to break.

Correct DNA origami folding from digested scaffold was confirmed by AFM imaging; see Figure 1. Freshly cleaved mica surfaces were coated by $0.01 \%$ poly-L-ornithine to facilitate origami immobilization. Imaging was carried out in intermittent contact mode.

AFM Sample Preparation. Clean glass cover slides and UV-ozone cleaned silicon nitride AFM cantilevers were covalently functionalized first with aminosilane and subsequently with $5 \mathrm{kDa}$ NHS-PEGmaleimide polymer linkers. Reduced sulfhydryl groups of DNA origami were coupled to the free maleimide groups on the cover slides by forming permanent thiol-ether bonds. ${ }^{74,94}$ Maleimide groups on the cantilevers were bound to coenzyme $\mathrm{A}$, and subsequently to a monovalent variant of StrepTactin (monoST) $)^{95,96}$ or a monomeric variant of streptavidin ${ }^{97}$ via Sfp phosphopantetheinyl transferase (SFP) with ybbR tags (amino acid sequence DSLEFIASKLA) for use as specific AFM pulling handles. ${ }^{98,99}$

SMFS Measurement Protocol. SMFS experiments were performed in TE buffer supplemented with magnesium at room temperature with custom-built instruments. Pulling velocities were set to 1 or $1.6 \mu \mathrm{m} \mathrm{s}^{-1}$. Cantilever and glass slide positioning was controlled by PID feedback loops during measurement. For analysis, curves with peaks at more than $150 \mathrm{~nm}$ extension and more than 30 $\mathrm{pN}$ force were sorted automatically, after thermal cantilever calibration, interferometric piezo calibration, and data conversion, similar to previous work. ${ }^{99-102}$

Simulations. We ran molecular dynamics (MD) simulations using oxDNA, a coarse-grained model whose basic unit is a rigid nucleotide that interacts with other nucleotides through stacking, hydrogenbonding, excluded volume, electrostatic and backbone potentials. An Andersen-like thermostat ${ }^{103}$ ensured diffusive particle motion in the canonical ensemble. Simulations were performed on GPUs and ranged

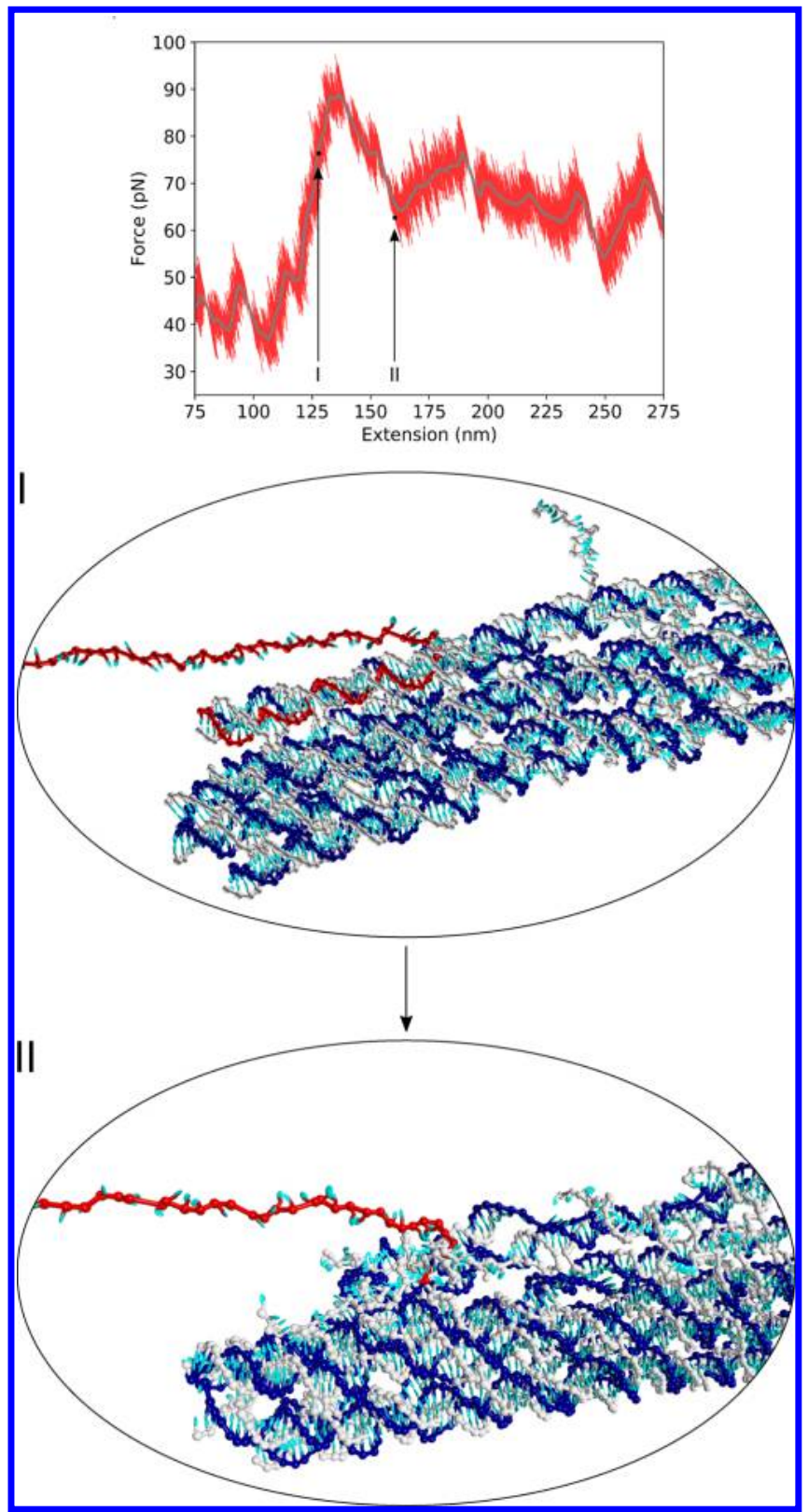

Figure 10. Structural illustration of the first large unfolding rip in a force-extension curve collected at $\dot{F}=3.2 \times 10^{8} \mathrm{pN} / \mathrm{s}$. The origin of the "strain-softening effect", in which subsequent unfolding rips occur at lower forces, can be seen in the images: once the first helix (red) completely unwinds between I and II, many of the multipledomain staples (white) attached to other helices (blue) have been compromised. Subsequent helices thus require lower forces to yield.

in duration from $O\left(10^{8}\right)$ to $O\left(10^{9}\right)$ steps. At the slowest pulling rate, the Rothemund tile simulation took $\sim 240$ days, the 10-helix bundle simulation took $\sim 30$ days, and the seven-tile biosensor simulation took $\sim 50$ days on a single GPU. Temperatures relevant to experiments were used: $20^{\circ} \mathrm{C}$ for the tile, $36^{\circ} \mathrm{C}$ for the helix bundle, ${ }^{64}$ and $30{ }^{\circ} \mathrm{C}$ for the seven-tile assembly; and we used a high salt concentration typical of origami studies, $\left[\mathrm{Na}^{+}\right]=0.5 \mathrm{M}$.

Sequence dependence did not significantly alter the unfolding behavior for the tile and was only incorporated into the tile simulations at $3.2 \times 10^{9} \mathrm{pN} / \mathrm{s}$. Simulations of the $10 \mathrm{HB}$ and seven-tile structures did not incorporate sequence dependence.

In all cases of harmonic pulling, the centers-of-mass of the scaffold end nucleotides were subjected to 3D harmonic traps. Scaffold end-to- 


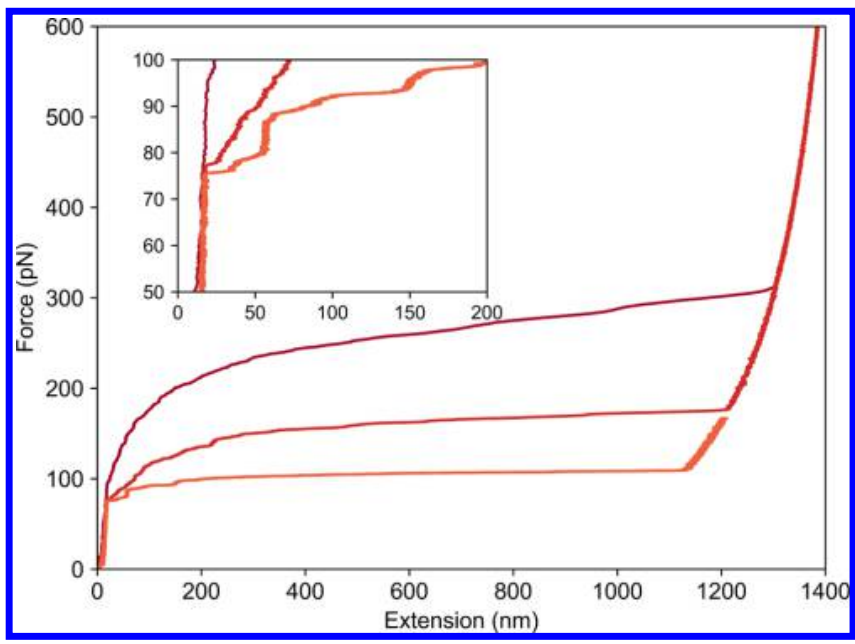

Figure 11. Simulated force-extension curves for the 10-helix bundle collected under a linearly increasing force protocol. Force loading rates decrease from top to bottom: $\dot{F}=3.2 \times 10^{8} \mathrm{pN} / \mathrm{s}, 3.2$ $\times 10^{7} \mathrm{pN} / \mathrm{s}, 3.2 \times 10^{6} \mathrm{pN} / \mathrm{s}$. The inset shows details of the initial rupture events preceding catastrophic unfolding. end separation along the axis separating the traps was measured and subtracted from the separation between trap centers to yield an "effective" trap displacement, which was multiplied by the stiffness of the traps in series to give the instantaneous force. For linear pulling, a constantly increasing tension was applied to scaffold end nucleotides.

Additional simulation details are provided in the Supporting Information.

\section{ASSOCIATED CONTENT}

\section{S Supporting Information}

The Supporting Information is available free of charge on the ACS Publications website at DOI: 10.1021/acsnano.8b01844.

Unfolding animation (MPG)

Unfolding animation (MPG)

Fuller details of experimental and simulation methods and additional simulation results (PDF)

\section{AUTHOR INFORMATION}

\section{Corresponding Author}

*E-mail: megan.engel@physics.ox.ac.uk.

ORCID

Megan C. Engel: 0000-0002-2598-9711

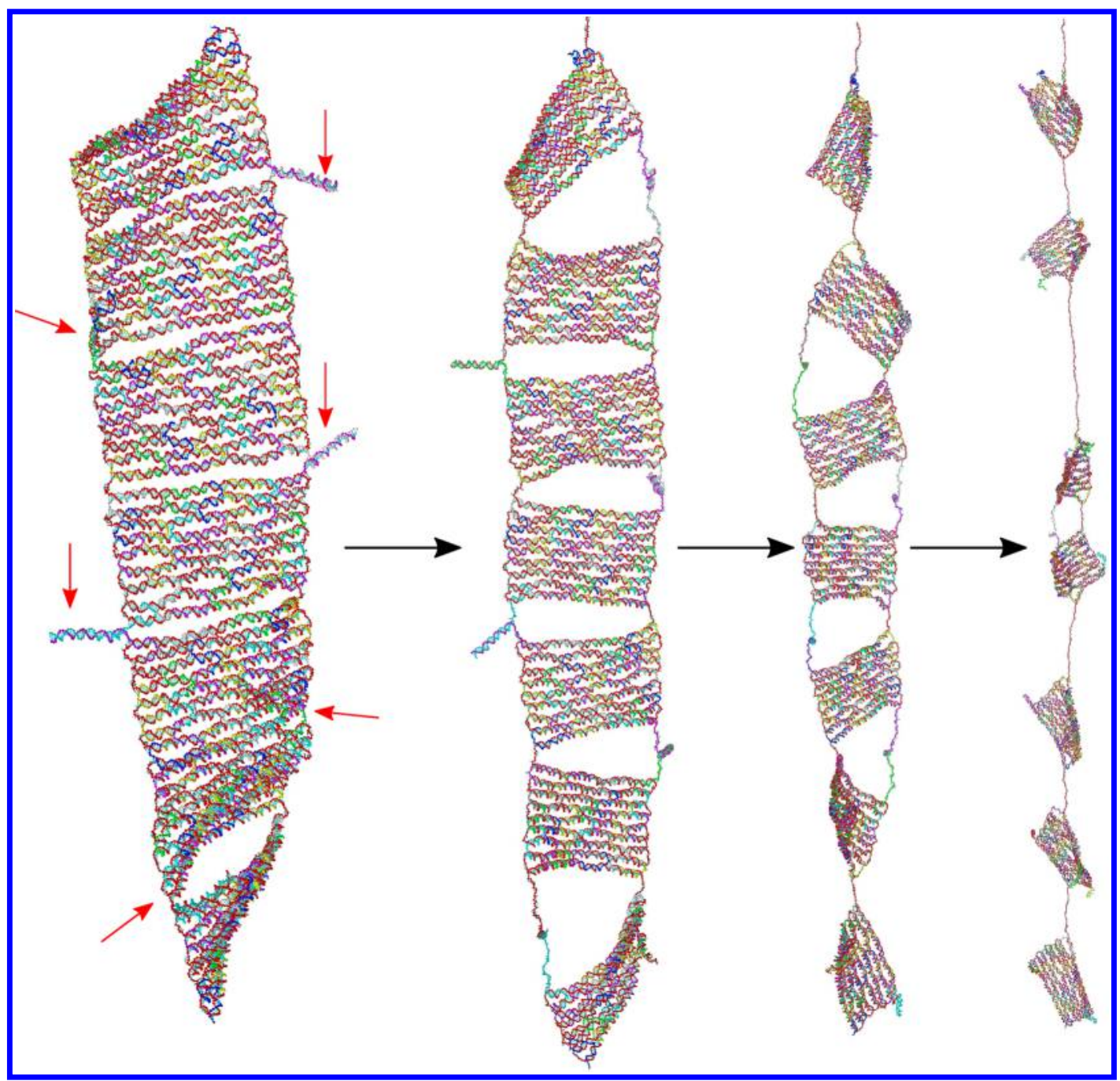

Figure 12. Structural snapshots illustrating the unfolding mechanism of the seven-tile biosensor of Koirala $e t$ al..$^{36}$ at a force-loading rate of $\dot{F}=$ $3.2 \times 10^{8} \mathrm{pN} / \mathrm{s}$. The duplex "locks" are labeled with red arrows in the first panel. The end-to-end length in the leftmost panel is $181 \mathrm{~nm}$, and in the rightmost panel is $475 \mathrm{~nm}$. As expected and experimentally observed, the locks open outermost to innermost. 
Tim Liedl: 0000-0002-0040-0173

Lorenzo Rovigatti: 0000-0001-5017-2829

Jonathan P. K. Doye: 0000-0002-2226-9524

\section{Notes}

The authors declare no competing financial interest.

\section{ACKNOWLEDGMENTS}

M.C.E. is grateful to the Canadian Natural Sciences and Engineering Research Council (NSERC) and the Rhodes Trust for financial support. The authors acknowledge the use of the University of Oxford Advanced Research Computing (ARC) facility (http://dx.doi.org/10.5281/zenodo.22558) and the resources provided by the "Cambridge Service for Data Driven Discovery" (CSD3) system operated by the University of Cambridge Research Computing Service funded by EPSRC Tier-2 capital grant EP/P020259/1. Part of this work has been supported through the Fraunhofer Attract project 601 683. L.R. acknowledges support from the European Commission through the Marie Skłodowska-Curie Fellowship No. 702298-DELTAS.

\section{REFERENCES}

(1) Smith, S. B.; Cui, Y.; Bustamante, C. Overstretching B-DNA: The Elastic Response of Individual Double-Stranded and Single-Stranded DNA Molecules. Science 1996, 271, 795-799.

(2) Bosco, A.; Camunas-Soler, J.; Ritort, F. Elastic Properties and Secondary Structure Formation of Single-Stranded DNA at Monovalent and Divalent Salt Conditions. Nucleic Acids Res. 2014, 42, 2064-2074.

(3) McIntosh, D. B.; Ribeck, N.; Saleh, O. A. Detailed Scaling Analysis of Low-Force Polyelectrolyte Elasticity. Phys. Rev. E 2009, 80, 041803.

(4) Essevaz-Roulet, B.; Bockelmann, U.; Heslot, F. Mechanical Separation of the Complementary Strands of DNA. Proc. Natl. Acad. Sci. U. S. A. 1997, 94, 11935-11940.

(5) Strunz, T.; Oroszlan, K.; Schäfer, R.; Güntherodt, H.-J. Dynamic Force Spectroscopy of Single DNA Molecules. Proc. Natl. Acad. Sci. U. S. A. 1999, 96, 11277-11282.

(6) Kühner, F.; Morfill, J.; Neher, R. A.; Blank, K.; Gaub, H. E. ForceInduced DNA Slippage. Biophvs. I. 2007, 92, 2491-2497.

(7) van Mameren, J.; Gross, P.; Farge, G.; Hooijman, P.; Modesti, M.; Falkenberg, M.; Wuite, G. J. L.; Peterman, E. J. G. Unraveling the Structure of DNA During Overstretching by Using Multicolor, SingleMolecule Fluorescence Imaging. Proc. Natl. Acad. Sci. U. S. A. 2009, 106, 18231-18236.

(8) Gross, P.; Laurens, N.; Oddershede, L. B.; Bockelmann, U.; Peterman, E. J. G.; Wuite, G. J. L. Quantifying How DNA Stretches, Melts and Changes Twist Under Tension. Nat. Phvs. 2011, 7, 731736.

(9) Romano, F.; Chakraborty, D.; Doye, J. P. K.; Ouldridge, T. E.; Louis, A. A. Coarse-grained simulations of DNA overstretching. J. Chem. Phys. 2013, 138, 085101.

(10) Forth, S.; Deufel, C.; Sheinin, M. Y.; Daniels, B.; Sethna, J. P.; Wang, M. D. Abrupt Buckling Transition Observed During the Plectoneme Formation of Individual DNA Molecules. Phys. Rev. Lett. 2008, 100, 148301.

(11) Nomidis, S. K.; Kriegel, F.; Vanderlinden, W.; Lipfert, J.; Carlon, E. Twist-Bend Coupling and the Torsional Response of DoubleStranded DNA. Phvs. Rev. Lett. 2017, 118, 217801.

(12) Zhang, Y.; McEwen, A.; Crothers, D.; Levene, S. Analysis of InVivo LacR-Mediated Gene Repression Based on the Mechanics of DNA Looping. PLoS One 2006, 1, e136.

(13) Goodman, S. D.; Nash, H. A. Functional Replacement of a Protein-Induced Bend in a DNA Recombination Site. Nature 1989, $341,251-254$.

(14) Bustamante, C.; Bryant, Z.; Smith, S. B. Ten Years of Tension: Single-Molecule DNA Mechanics. Nature 2003, 421, 423-427.
(15) Goktas, M.; Blank, K. G. Molecular Force Sensors: From Fundamental Concepts Toward Applications in Cell Biology. Adv. Mater. Interfaces 2017, 4, 1600441.

(16) Wang, X.; Ha, T. Defining Single Molecular Forces Required to Activate Integrin and Notch Signaling. Science 2013, 340, 991-994.

(17) Blakely, B. L.; Dumelin, C. E.; Trappmann, B.; McGregor, L. M.; Choi, C. K.; Anthony, P. C.; Duesterberg, V. K.; Baker, B. M.; Block, S. M.; Liu, D. R.; Chen, C. S. A DNA-based Molecular Probe for Optically Reporting Cellular Traction Forces. Nat. Nat. Methods 2014, 11, 1229-1232.

(18) Cocco, S.; Monasson, R.; Marko, J. F. Force and Kinetic Barriers to Unzipping of the DNA Double Helix. Proc. Natl. Acad. Sci. U. S. A. 2001, 98, 8608-8613.

(19) Mosayebi, M.; Louis, A. A.; Doye, J. P. K.; Ouldridge, T. E. Force-Induced Rupture of a DNA Duplex: From Fundamentals to Force Sensors. ACS Nano 2015, 9, 11993-12003.

(20) Evans, E.; Ritchie, K. Dynamic Strength of Molecular Adhesion Bonds. Biophvs. I. 1997, 72, 1541-1555.

(21) Hatch, K.; Danilowicz, C.; Coljee, V.; Prentiss, M. Demonstration That the Shear Force Required to Separate Short Double-Stranded DNA Does Not Increase Significantly With Sequence Length for Sequences Longer Than 25 Base Pairs. Phys. Rev. E 2008, 78, 011920.

(22) Neher, R. A.; Gerland, U. DNA as a Programmable Viscoelastic Nanoelement. Biophvs. I. 2005, 89, 3846-3855.

(23) Williams, M. C.; Wenner, J. R.; Rouzina, I.; Bloomfield, V. A. Entropy and Heat Capacity of DNA Melting From Temperature Dependence of Single Molecule Stretching. Biophys. J. 2001, 80, 1932-1939.

(24) Wenner, J. R.; Williams, M. C.; Rouzina, I.; Bloomfield, V. A. Salt Dependence of the Elasticity and Overstretching Transition of Single DNA Molecules. Biophvs. I. 2002, 82, 3160-3169.

(25) Fu, H.; Chen, H.; Koh, C. G.; Lim, C. T. Effects of Magnesium Salt Concentrations on B-DNA Overstretching Transition. Eur. Phys. J. E: Soft Matter Biol. Phys. 2009, 29, 45-49.

(26) Huguet, J. M.; Bizarro, C. V.; Forns, N.; Smith, S. B.; Bustamante, C.; Ritort, F. Single-Molecule Derivation of Salt Dependent Base-Pair Free Energies in DNA. Proc. Natl. Acad. Sci. U. S. A. 2010, 107, 15431-15436.

(27) Gaub, H. E.; Rief, M.; Clausen-Schaumann, H. SequenceDependent Mechanics of Single DNA Molecules. Nat. Struct. Biol. 1999, 6, 346-349.

(28) Seeman, N. C. Nanomaterials Based on DNA. Annu. Rev. Biochem. 2010, 79, 65-87.

(29) Jones, M. R.; Seeman, N. C.; Mirkin, C. A. Programmable Materials and the Nature of the DNA Bond. Science 2015, 347, 1260901.

(30) Fu, T. J.; Seeman, N. C. DNA Double-Crossover Molecules. Biochemistry 1993, 32, 3211-3220.

(31) Liu, D.; Wang, M.; Deng, Z.; Walulu, R.; Mao, C. Tensegrity:? Construction of Rigid DNA Triangles With Flexible Four-Arm DNA Iunctions. I. Am. Chem. Soc. 2004, 126, 2324-2325.

(32) He, Y.; Ye, T.; Su, M.; Zhang, C.; Ribbe, A. E.; Jiang, W.; Mao, C. Hierarchical Self-Assembly of DNA Into Symmetric Supramolecular Polvhedra. Nature 2008, 452, 198-201.

(33) Mathieu, F.; Liao, S.; Kopatsch, J.; Wang, T.; Mao, C.; Seeman, N. C. Six-Helix Bundles Designed From DNA. Nano Lett. 2005, 5, 661-665.

(34) Rothemund, P. W. K. Folding DNA to Create Nanoscale Shapes and Patterns. Nature 2006, 440, 297-302.

(35) Bell, N. A. W.; Engst, C. R.; Ablay, M.; Divitini, G.; Ducati, C.; Liedl, T.; Keyser, U. F. DNA Origami Nanopores. Nano Lett. 2012, 12, 512-517.

(36) Koirala, D.; Shrestha, P.; Emura, T.; Hidaka, K.; Mandal, S.; Endo, M.; Sugiyama, H.; Mao, H. Single-Molecule Mechanochemical Sensing Using DNA Origami Nanostructures. Angew. Chem. 2014, $126,8275-8279$.

(37) Andersen, E. S.; Dong, M.; Nielsen, M. M.; Jahn, K.; Subramani, R.; Mamdouh, W.; Golas, M. M.; Sander, B.; Stark, H.; Oliveira, C. L. 
P.; Pedersen, J. S.; Birkedal, V.; Besenbacher, F.; Gothelf, K. V.; Kjems, J. r. Self-Assembly of a Nanoscale DNA Box With a Controllable Lid. Nature 2009, 459, 73-76.

(38) Pinheiro, A. V.; Han, D.; Shih, W. M.; Yan, H. Challenges and Opportunities for Structural DNA Nanotechnology. Nat. Nanotechnol. 2011, 6, 763-772.

(39) Yin, P.; Hariadi, R. F.; Sahu, S.; Choi, H. M. T.; Park, S. H.; LaBean, T. H.; Reif, J. H. Programming DNA Tube Circumferences. Science 2008, 321, 824-826.

(40) Wei, B.; Dai, M.; Yin, P. Complex Shapes Self-Assembled From Single-Stranded DNA Tiles. Nature 2012, 485, 623.

(41) Ke, Y.; Ong, L. L.; Shih, W. M.; Yin, P. Three-Dimensional Structures Self-Assembled From DNA Bricks. Science 2012, 338, $1177-1183$.

(42) Sa-Ardyen, P.; Vologodskii, A. V.; Seeman, N. C. The Flexibility of DNA Double Crossover Molecules. Biophys. I. 2003, 84, 38293837.

(43) Kauert, D. J.; Kurth, T.; Liedl, T.; Seidel, R. Direct Mechanical Measurements Reveal the Material Properties of Three-Dimensional DNA Origami. Nano Lett. 2011, 11, 5558-5563.

(44) Wang, T.; Schiffels, D.; Martinez Cuesta, S.; Kuchnir Fygenson, D.; Seeman, N. C. Design and Characterization of 1D Nanotubes and 2D Periodic Arrays Self-Assembled From DNA Multi-Helix Bundles. J. Am. Chem. Soc. 2012, 134, 1606-1616.

(45) Schiffels, D.; Liedl, T.; Fygenson, D. K. Nanoscale Structure and Microscale Stiffness of DNA Nanotubes. ACS Nano 2013, 7, 67006710

(46) Yoo, J.; Aksimentiev, A. In Situ Structure and Dynamics of DNA Origami Determined Through Molecular Dynamics Simulations. Proc. Natl. Acad. Sci. U. S. A. 2013, 110, 20099-20104.

(47) Slone, S. M.; Li, C.-Y.; Yoo, J.; Aksimentiev, A. Molecular Mechanics of DNA Bricks: In Situ Structure, Mechanical Properties and Ionic Conductivity. New I. Phvs. 2016, 18, 055012.

(48) Castro, C. E.; Kilchherr, F.; Kim, D.-N.; Shiao, E. L.; Wauer, T.; Wortmann, P.; Bathe, M.; Dietz, H. A Primer to Scaffolded DNA Origami. Nat. Methods 2011, 8, 221-229.

(49) Kim, D.-N.; Kilchherr, F.; Dietz, H.; Bathe, M. Quantitative Prediction of 3D Solution Shape and Flexibility of Nucleic Acid Nanostructures. Nucleic Acids Res. 2012, 40, 2862-2868.

(50) Reshetnikov, R. V.; Stolyarova, A. V.; Zalevsky, A. O.; Panteleev, D. Y.; Pavlova, G. V.; Klinov, D. V.; Golovin, A. V.; Protopopova, A. D. A Coarse-Grained Model for DNA Origami. Nucleic Acids Res. 2018, 46, 1102-1112.

(51) Schreck, J. S.; Romano, F.; Zimmer, M. H.; Louis, A. A.; Doye, J. P. K. Characterizing DNA Star-Tile-Based Nanostructures Using a Coarse-Grained Model. ACS Nano 2016, 10, 4236-4247.

(52) Ke, Y.; Lindsay, S.; Chang, Y.; Liu, Y.; Yan, H. Self-Assembled Water-Soluble Nucleic Acid Probe Tiles for Label-Free RNA Hybridization Assays. Science 2008, 319, 180-183.

(53) Gu, H.; Yang, W.; Seeman, N. C. DNA Scissors Device Used to Measure MutS Binding to DNA Mis-Pairs. J. Am. Chem. Soc. 2010, 132, 4352-4357.

(54) Iwaki, M.; Wickham, S. F.; Ikezaki, K.; Yanagida, T.; Shih, W. M. A Programmable DNA Origami Nanospring That Reveals ForceInduced Adjacent Binding of Myosin VI Heads. Nat. Commun. 2016, 7,13715 .

(55) Funke, J. J.; Ketterer, P.; Lieleg, C.; Schunter, S.; Korber, P.; Dietz, H. Uncovering the Forces Between Nucleosomes Using DNA Origami. Sci. Adv. 2016, 2, No. e1600974.

(56) Nickels, P. C.; Wünsch, B.; Holzmeister, P.; Bae, W.; Kneer, L. M.; Grohmann, D.; Tinnefeld, P.; Liedl, T. Molecular Force Spectroscopy With a DNA Origami-based Nanoscopic Force Clamp. Science 2016, 354, 305-307.

(57) Liedl, T.; Hogberg, B.; Tytell, J.; Ingber, D. E.; Shih, W. M. SelfAssembly of Three-Dimensional Prestressed Tensegrity Structures From DNA. Nat. Nanotechnol. 2010, 5, 520-524.

(58) Simmel, S. S.; Nickels, P. C.; Liedl, T. Wireframe and Tensegrity DNA Nanostructures. Acc. Chem. Res. 2014, 47, 1691-1699.
(59) Marras, A. E.; Zhou, L.; Su, H.-J.; Castro, C. E. Programmable Motion of DNA Origami Mechanisms. Proc. Natl. Acad. Sci. U. S. A. 2015, 112, 713-718.

(60) Bell, N. A. W.; Engst, C. R.; Ablay, M.; Divitini, G.; Ducati, C.; Liedl, T.; Keyser, U. F. DNA Origami Nanopores. Nano Lett. 2012, 12, 512-517.

(61) Wei, R; Martin, T. G.; Rant, U.; Dietz, H. DNA Origami Gatekeepers for Solid-State Nanopores. Angew. Chem., Int. Ed. 2012, 51, 4864-4867.

(62) Schuldt, C.; Schnauß, J.; Händler, T.; Glaser, M.; Lorenz, J.; Golde, T.; Käs, J. A.; Smith, D. M. Tuning Synthetic Semiflexible Networks by Bending Stiffness. Phvs. Rev. Lett. 2016, 117, 197801.

(63) Shrestha, P.; Emura, T.; Koirala, D.; Cui, Y.; Hidaka, K.; Maximuck, W. J.; Endo, M.; Sugiyama, H.; Mao, H. Mechanical Properties of DNA Origami Nanoassemblies Are Determined by Holliday Junction Mechanophores. Nucleic Acids Res. 2016, 44, 65746582.

(64) Bae, W.; Kim, K.; Min, D.; Ryu, J.-K.; Hyeon, C.; Yoon, T.-Y. Programmed Folding of DNA Origami Structures Through SingleMolecule Force Control. Nat. Commun. 2014, 5, 5654.

(65) Ouldridge, T. E.; Louis, A. A.; Doye, J. P. K. Structural, Mechanical, and Thermodynamic Properties of a Coarse-GCrained DNA Model. I. Chem. Phvs. 2011, 134, 085101.

(66) Sulc, P.; Romano, F.; Ouldridge, T. E.; Rovigatti, L.; Doye, J. P. K.; Louis, A. A. Sequence-Dependent Thermodynamics of a CoarseGrained DNA Model. J. Chem. Phys. 2012, 137, 135101.

(67) Snodin, B. E. K.; Randisi, F.; Mosayebi, M.; Šulc, P.; Schreck, J. S.; Romano, F.; Ouldridge, T. E.; Tsukanov, R.; Nir, E.; Louis, A. A.; Doye, J. P. K. Introducing Improved Structural Properties and Salt Dependence Into a Coarse-Grained Model of DNA. J. Chem. Phys. 2015, 142, 234901.

(68) Sharma, R.; Schreck, J. S.; Romano, F.; Louis, A. A.; Doye, J. P. $\mathrm{K}$. Characterizing the Motion of Jointed DNA Nanostructures Using a Coarse-Grained Model. ACS Nano 2017, 11, 12426-12435.

(69) Romano, F.; Chakraborty, D.; Doye, J. P. K.; Ouldridge, T. E.; Louis, A. A. Coarse-Grained Simulations of DNA Overstretching. J. Chem. Phys. 2013, 138, 085101.

(70) Matek, C.; Ouldridge, T. E.; Doye, J. P. K.; Louis, A. A. Plectoneme Tip Bubbles: Coupled Denaturation and Writhing in Supercoiled DNA. Sci. Rep. 2015, 5, 7655.

(71) Matek, C.; Ouldridge, T.; Levy, A.; Doye, J.; Louis, A. DNA Cruciform Arms Nucleate Through a Correlated but Asynchronous Cooperative Mechanism. I. Phys. Chem. B 2012, 116, 11616-11625.

(72) Depa, P.; Chen, C.; Maranas, J. K. Why Are Coarse-Grained Force Fields Too Fast? A Look at Dynamics of Four Coarse-Grained Polvmers. I. Chem. Phvs. 2011, 134, 014903.

(73) Padding, J. T.; Louis, A. A. Hydrodynamic Interactions and Brownian Forces in Colloidal Suspensions: Coarse-Graining Over Time and Length Scales. Phys. Rev. E 2006, 74, 031402.

(74) Jobst, M. A.; Milles, L. F.; Schoeler, C.; Ott, W.; Fried, D. B.; Bayer, E. A.; Gaub, H. E.; Nash, M. A. Resolving Dual Binding Conformations of Cellulosome Cohesin-Dockerin Complexes Using Single-Molecule Force Spectroscopy. eLife 2015, 4, 180-183.

(75) Schoeler, C.; Verdorfer, T.; Gaub, H. E.; Nash, M. A. Biasing Effects of Receptor-Ligand Complexes on Protein-Unfolding Statistics. Phys. Rev. E: Stat. Phys., Plasmas, Fluids, Relat. Interdiscip. Top. 2016, 94, 042412.

(76) Kramers, H. Brownian Motion in a Field of Force and the Diffusion Model of Chemical Reactions. Phvsica 1940, 7, 284-304.

(77) Ray, C.; Brown, J. R.; Akhremitchev, B. B. Correction of Systematic Errors in Single-Molecule Force Spectroscopy With Polymeric Tethers by Atomic Force Microscopy. J. Phys. Chem. B 2007, 111, 1963-1974.

(78) Neuert, G.; Albrecht, C.; Pamir, E.; Gaub, H. Dynamic Force Spectroscopy of the Digoxigenin Antibody Complex. FEBS Lett. 2006, 580, 505-509.

(79) Alemany, A.; Ritort, F. Determination of the elastic properties of short ssDNA molecules by mechanically folding and unfolding DNA hairpins. Biopolvmers 2014, 101, 1193-1199. 
(80) Woodside, M. T.; Block, S. M. Reconstructing Folding Energy Landscapes by Single-Molecule Force Spectroscopy. Annu. Rev. Biophys. 2014, 43, 19-39.

(81) Dudko, O. K.; Hummer, G.; Szabo, A. Intrinsic Rates and Activation Free Energies From Single-Molecule Pulling Experiments. Phvs. Rev. Lett. 2006, 96, 108101.

(82) Bullerjahn, J. T.; Sturm, S.; Kroy, K. Theory of Rapid Force Spectroscopy. Nat. Commun. 2014, 5, 4463.

(83) Bell, G. Models for the Specific Adhesion of Cells to Cells. Science 1978, 200, 618-627.

(84) Hummer, G.; Szabo, A. Kinetics From Nonequilibrium SingleMolecule Pulling Experiments. Biophvs. I. 2003, 85, 5-15.

(85) Getfert, S.; Reimann, P. Hidden Multiple Bond Effects in Dynamic Force Spectroscopy. Biophys. I. 2012, 102, 1184-1193.

(86) Ritchie, D. B.; Foster, D. A. N.; Woodside, M. T. Programmed -1 Frameshifting Efficiency Correlates With RNA Pseudoknot Conformational Plasticity, Not Resistance to Mechanical Unfolding. Proc. Natl. Acad. Sci. U. S. A. 2012, 109, 16167-16172.

(87) Strackharn, M.; Stahl, S. W.; Puchner, E. M.; Gaub, H. E. Functional Assembly of Aptamer Binding Sites by Single-Molecule Cut-And-Paste. Nano Lett. 2012, 12, 2425-2428.

(88) Scargle, J. D. Studies in Astronomical Time Series Analysis. II Statistical Aspects of Spectral Analysis of Unevenly Spaced Data. Astrophvs. I. 1982, 263, 835-853.

(89) Fugmann, S.; Sokolov, I. M. Scaling of the Rupture Dynamics of Polymer Chains Pulled at One End at a Constant Rate. Phys. Rev. E 2009, 79, 021803.

(90) Puchner, E. M.; Gaub, H. E. Single-Molecule Mechanoenzymatics. Annu. Rev. Biophvs. 2012, 41, 497-518.

(91) Douglas, S. M.; Marblestone, A. H.; Teerapittayanon, S.; Vazquez, A.; Church, G. M.; Shih, W. M. Rapid Prototyping of 3D DNA-origami Shapes With caDNAno. Nucleic Acids Res. 2009, 37, 5001-5006.

(92) Sajfutdinow, M.; Uhlig, K.; Prager, A.; Schneider, C.; Abel, B.; Smith, D. M. Nanoscale Patterning of Self-Assembled Monolayer (SAM)-Functionalised Substrates With Single Molecule Contact Printing. Nanoscale 2017, 9, 15098-15106.

(93) Stahl, E.; Martin, T. G.; Praetorius, F.; Dietz, H. Facile and Scalable Preparation of Pure and Dense DNA Origami Solutions. Angew. Chem. Int. Ed. 2014, 53, 12735-12740.

(94) Jobst, M. A.; Schoeler, C.; Malinowska, K.; Nash, M. A. Investigating Receptor-Ligand Systems of the Cellulosome With AFMbased Single-Molecule Force Spectroscopy. J. Visualized Exp. 2013, 50950.

(95) Baumann, F.; Bauer, M. S.; Milles, L. F.; Alexandrovich, A.; Gaub, H. E.; Pippig, D. A. Monovalent Strep-Tactin for Strong and Site-Specific Tethering in Nanospectroscopy. Nat. Nanotechnol. 2015, $11,89-94$.

(96) Erlich, K. R.; Baumann, F.; Pippig, D. A.; Gaub, H. E. Strep-Tag II and Monovalent Strep-Tactin as Novel Handles in Single-Molecule Cut-And-Paste. Small Methods 2017, 1, 1700169.

(97) Bauer, M. S.; Milles, L. F.; Sedlak, S. M.; Gaub, H. E. Monomeric Streptavidin: A Versatile Regenerative Handle for Force Spectroscopy. bioRxiv 2018, DOI: 10.1101/276444.

(98) Pippig, D. A.; Baumann, F.; Strackharn, M.; Aschenbrenner, D.; Gaub, H. E. Protein-DNA Chimeras for Nano Assembly. ACS Nano 2014, 8, 6551-6555.

(99) Otten, M.; Ott, W.; Jobst, M. A.; Milles, L. F.; Verdorfer, T.; Pippig, D. A.; Nash, M. A.; Gaub, H. E. From Genes to Protein Mechanics on a Chip. Nat. Methods 2014, 11, 1127-1130.

(100) Ott, W.; Jobst, M. A.; Bauer, M. S.; Durner, E.; Milles, L. F.; Nash, M. A.; Gaub, H. E. Elastin-Like Polypeptide Linkers for SingleMolecule Force Spectroscopy. ACS Nano 2017, 11, 6346-6354.

(101) Hutter, J. L.; Bechhoefer, J. Calibration of Atomic-Force Microscope Tips. Rev. Sci. Instrum. 1993, 64, 1868-1873.

(102) Cook, S. M.; Scäffer, T. E.; Chynoweth, K. M.; Wigton, M.; Simmonds, R. W.; Lang, K. M. Practical Implementation of Dynamic Methods for Measuring Atomic Force Microscope Cantilever Spring Constants. Nanotechnology 2006, 17, 2135-2145.
(103) Russo, J.; Tartaglia, P.; Sciortino, F. Reversible Gels of Patchy Particles: Role of the Valence. I. Chem. Phys. 2009, 131, 014504. 